\title{
CANONICAL TRACES AND DIRECTLY FINITE LEAVITT PATH ALGEBRAS
}

\author{
LIA VA ̌
}

\begin{abstract}
Motivated by the study of traces on graph $C^{*}$-algebras, we consider traces (additive, central maps) on Leavitt path algebras, the algebraic counterparts of graph $C^{*}$ algebras. In particular, we consider traces which vanish on nonzero graded components of a Leavitt path algebra and refer to them as canonical since they are uniquely determined by their values on the vertices.

A desirable property of a $\mathbb{C}$-valued trace on a $C^{*}$-algebra is that the trace of an element of the positive cone is nonnegative. We adapt this property to traces on a Leavitt path algebra $L_{K}(E)$ with values in any involutive ring. We refer to traces with this property as positive. If a positive trace is injective on positive elements, we say that it is faithful. We characterize when a canonical, $K$-linear trace is positive and when it is faithful in terms of its values on the vertices. As a consequence, we obtain a bijective correspondence between the set of faithful, gauge invariant, $\mathbb{C}$-valued (algebra) traces on $L_{\mathbb{C}}(E)$ of a countable graph $E$ and the set of faithful, semifinite, lower semicontinuous, gauge invariant (operator theory) traces on the corresponding graph $C^{*}$-algebra $C^{*}(E)$.

With the direct finite condition (i.e $x y=1$ implies $y x=1$ ) for unital rings adapted to rings with local units, we characterize directly finite Leavitt path algebras as exactly those having the underlying graphs in which no cycle has an exit. Our proof involves consideration of "local" Cohn-Leavitt subalgebras of finite subgraphs. Lastly, we show that, while related, the class of locally noetherian, the class of directly finite, and the class of Leavitt path algebras which admit a faithful trace are different in general.
\end{abstract}

\section{INTRODUCTION}

Throughout their existence, many operator theory concepts have been subject to "algebraization" - the study of algebraic counterparts of operator theory concepts using algebraic methods alone. Regular rings, Baer rings, and their numerous generalizations have all been created by algebraization of some operator theory concepts. Recently, Leavitt path algebras have joined this list as algebraic counterparts of graph $C^{*}$-algebras and many properties of graph $C^{*}$-algebras have been formulated for Leavitt path algebras and proven using solely algebraic methods. Our interest in traces is greatly inspired by their relevance in the study of noncommutative geometry of graph $C^{*}$-algebras from 14 .

The class of all traces, i.e. additive and central maps, on a Leavitt path algebra is a rather large class. After some preliminaries, in section 2, we restrict our attention to those traces that vanish on nonzero graded components of a Leavitt path algebra and refer to them as canonical traces (Definition 2.5). Such traces are canonical in the sense that they are completely determined by the values on the vertices. In particular, we show that every graph trace (a map on the vertices of the underlying graph which agrees with the (CK2)

2000 Mathematics Subject Classification. 16S99, 16W99, 16W10, 16P99.

Key words and phrases. Trace, Leavitt path algebra, directly finite, involution, graph trace, gauge invariant, positive, faithful, Cohn-Leavitt algebra. 
axiom of Leavitt path algebras) uniquely extends to a canonical trace (Proposition 2.7). A canonical trace is gauge invariant and, if the characteristic of the underlying field is zero, the converse is true as well (Proposition 2.4).

In operator theory, a desirable property of a $\mathbb{C}$-valued trace on a $C^{*}$-algebra is that the trace of an element of the positive cone is nonnegative. The algebraic version of this property for a trace $t: R \rightarrow T$, where $R$ and $T$ are involutive rings, is that the trace of a positive element of $R$ (a finite sum of elements of the form $x x^{*}$ ) is a positive element of $T$. If a trace has this property we say it is positive. A positive trace is faithful if the trace of a nonzero, positive element is nonzero and positive.

Given a graph $E$ and a field $K$, consider the Leavitt path algebra $L_{K}(E)$ of $E$ over $K$. [12, Proposition 29] lists some necessary conditions, given in terms of trace values on the vertices of $E$, for a trace on $L_{K}(E)$ to be positive and faithful. In [12, it is shown that these conditions are not sufficient. In section $[3$, we prove that conditions (1)-(3) of [12, Proposition 29] are sufficient for a canonical, $K$-linear trace on $L_{K}(E)$ to be positive (Theorem 3.4 ). If a canonical, $K$-linear trace on $L_{K}(E)$ has values in a positive definite algebra, conditions (1)(4) are sufficient for this trace to be faithful (Theorem 3.5). Theorems 3.4 and 3.5 imply that any positive graph trace on $E$ uniquely extends to a positive, canonical, $K$-linear trace on $L_{K}(E)$ and, any faithful graph trace on $E$ with values in a positive definite algebra uniquely extends to a faithful, canonical, $K$-linear trace on $L_{K}(E)$ (Theorem 3.6).

Let $\mathbb{C}$ denote the field of complex numbers with the complex-conjugate involution. [14, Proposition 3.9] shows that there is a bijective correspondence between the set of faithful, $\mathbb{C}$ valued graph traces on a countable, row-finite graph $E$ and the set of faithful, semifinite, lower semicontinuous, gauge invariant, $\mathbb{C}$-valued traces (in the operator theory sense) on the graph $C^{*}$-algebra $C^{*}(E)$. We show that the two sets above are also in a bijective correspondence with the set of faithful, gauge invariant, $\mathbb{C}$-linear, $\mathbb{C}$-valued traces on the Leavitt path algebra $L_{\mathbb{C}}(E)$ and that it is not necessary to require that $E$ is row-finite (Corollary 3.8).

The main goal of the second part of the paper (section 4) is to characterize directly finite Leavitt path algebras by properties of the underlying graph. Recall that a unital ring is directly finite if $x y=1$ implies that $y x=1$ for all $x$ and $y$. We say that a ring with local units is directly finite if for every $x, y$ and an idempotent $u$ such that $x u=u x=x$ and $y u=u y=y$, we have that $x y=u$ implies $y x=u$. Inspired by results of Ara and Goodearl in [3], we note that consideration of finitely many elements of a Leavitt path algebra can be reduced to their consideration as elements of a Cohn-Leavitt subalgebra of a finite subgraph. We show that the Cohn-Leavitt path algebra of a finite graph $E$ is directly finite if and only if no cycle of $E$ has an exit and the (CK2) axiom holds for all vertices of the cycles. Using this result, we show that a Leavitt path algebra of a graph $E$ is directly finite if and only if no cycle of $E$ has an exit (Theorem 4.12).

Cohn-Leavitt algebras encompass both Cohn path algebras and Leavitt path algebras and can be viewed as algebraic counterparts of relative graph $C^{*}$-algebras. We adapt our previous results, Theorems 3.4, 3.5, and 3.6 to Cohn-Leavitt algebras (Propositions 4.4 and 4.6), and use our characterization of directly finite Leavitt path algebras to show that a Cohn path algebra $C_{K}(E)$ is directly finite if and only if $E$ is acyclic (Corollary 4.13). We also note that the properties that $L_{K}(E)$ is locally noetherian and that $L_{K}(E)$ admits a faithful trace are independent, both imply that $L_{K}(E)$ is directly finite and that both implications are strict (Examples 4.14, 4.15, and 4.16). We conclude the paper by considering an open problem (4.17). 


\section{Positive, faithful, and canonical traces on Leavitt path algebras}

Throughout the paper, all rings are assumed to be associative, but not necessarily unital. The notation $\delta_{a, b}$ is used to denote 1 if $a=b$ and 0 if $a \neq b$ for any set $A$ and $a, b$ in $A$. We start by recalling a few general definitions and establishing some preliminary results.

Let $R$ and $T$ be rings. A map $t: R \rightarrow T$ is central if $t(x y)=t(y x)$ for all $x, y \in R$ and it is a $T$-valued trace on $R$ if $t$ is an additive, central map. If $R$ and $T$ are $C$-algebras, for some commutative ring $C$, then the trace $t$ is $C$-linear if $t(c x)=\operatorname{ct}(x)$ for all $x \in R$ and $c \in C$.

The standard trace on a matrix ring over a commutative ring $C$ is an example of a $C$ linear, $C$-valued trace. If $G$ is a group, the Kaplansky trace and the augmentation map on the group ring $C G$ are also examples of $C$-linear, $C$-valued traces.

Recall that an involution on a ring $R$ is an additive map $*: R \rightarrow R$ such that $(x y)^{*}=y^{*} x^{*}$ and $\left(x^{*}\right)^{*}=x$ for all $x, y \in R$. In this case $R$ is called an involutive ring or a $*$-ring. If $R$ is also a $C$-algebra for some commutative, involutive ring $C$, then $R$ is a $*$-algebra if $(a x)^{*}=a^{*} x^{*}$ for $a \in C$ and $x \in R$.

An element of a *-ring $R$ is positive if it is a finite sum of elements of the form $x x^{*}$ for $x \in R$. The notation $x>0$ usually denotes positive elements. We abuse this notation slightly and denote the fact that $x$ is positive element by $x \geq 0$. If $x$ is positive and nonzero, we write $x>0$. One may argue that we should refer to positive elements as nonnegative instead. Although this may be a valid point, we continue to use the terminology which is well established in operator theory and keep referring to such elements as positive.

An involution $*$ on $R$ is positive definite if, for all $x_{1}, \ldots, x_{n} \in R, \sum_{i=1}^{n} x_{i} x_{i}^{*}=0$ implies $x_{i}=0$ for each $i=1, \ldots, n$ and it is proper if this condition holds for $n=1$. A $*$-ring with a positive definite (proper) involution is referred to as positive definite (proper). By [9, Exercise 9A, sec. 13], a $*$-ring $R$ is positive definite if and only $R$ is proper and the conditions

$$
x \geq 0, y \geq 0 \text {, and } x+y=0 \text { imply } x=y=0 .
$$

The relation $\geq$ extends to all elements of a $*$-ring $R$ by

$$
x \geq y \text { if and only if } x-y \geq 0 \text {. }
$$

This relation is always reflexive and transitive (see [9, Section 50]). The antisymmetry holds if $R$ is positive definite.

Let $R$ and $T$ be $*$-rings and $t: R \rightarrow T$ be an additive map.

The map $t$ is positive if $t(x) \geq 0$ for all $x \in R$ with $x \geq 0$ (equivalently $t\left(x x^{*}\right) \geq 0$ for all $x \in R$ ).

The map $t$ is faithful if $t(x)>0$ for all $x \in R$ with $x>0$ (equivalently $t$ is positive and $x \geq 0$ and $t(x)=0$ imply $x=0$ ).

The following lemma further characterizes faithful, additive maps with values in a positive definite $*$-ring.

Lemma 2.1. Let $R$ and $T$ be *-rings, $T$ be positive definite, and $t: R \rightarrow T$ be any positive, additive map. The following are equivalent.

(1) The map $t$ is faithful.

(2) $x \geq 0, y \geq 0$ and $t(x+y)=0$ imply $x=y=0$ for all $x, y \in R$.

Conditions (3) and (4) below imply (1) and (2). If $R$ is proper, then (1) and (2) are equivalent to $(3)$ and (4).

(3) $t\left(x x^{*}+y y^{*}\right)=0$ implies $x=y=0$ for all $x, y \in R$. 
(4) $t\left(x x^{*}\right)=0$ implies $x=0$ for all $x \in R$.

Proof. To prove that (1) implies (2), let $x \geq 0, y \geq 0$ and $t(x+y)=0$ for $x, y \in R$. Since $t$ is positive $t(x) \geq 0$ and $t(y) \geq 0$. Since $T$ is positive definite $t(x)+t(y)=t(x+y)=0$ implies that $t(x)=t(y)=0$. Then $x=y=0$ by faithfulness of $t$.

Condition (2) with $y=0$ implies condition (1) and condition (3) with $y=0$ implies condition (4).

Condition (4) implies (1). Indeed, if $x \geq 0$ and $t(x)=0$, then $x=\sum_{i=1}^{n} a_{i} a_{i}^{*}$ for some $a_{i} \in R$ and $0=t\left(\sum_{i=1}^{n} a_{i} a_{i}^{*}\right)=\sum_{i=1}^{n} t\left(a_{i} a_{i}^{*}\right)$. Since $T$ is positive definite and $t$ is positive, this implies that $t\left(a_{i} a_{i}^{*}\right)=0$ for all $i=1, \ldots, n$. Then $a_{i}=0$ for all $i=1, \ldots, n$ by condition (4). Thus $x=0$.

Assuming now that $R$ is proper and that condition (2) holds, let us show (3). If $t\left(x x^{*}+\right.$ $\left.y y^{*}\right)=0$, then condition (2) implies that $x x^{*}=0$ and $y y^{*}=0$. Then $x=y=0$ by properness of $R$.

Since any $C^{*}$-algebra is proper $([9$, page 11$])$ and $\mathbb{C}$ is positive definite when equipped with the complex-conjugate involution, a $\mathbb{C}$-valued, additive, and positive map on a $C^{*}$-algebra can be defined to be faithful using any of the conditions (1)-(4). In fact, in operator theory texts, either condition (3) or condition (4) are frequently used when defining a faithful trace.

We review the definition of a Leavitt path algebra now. Let $E=\left(E^{0}, E^{1}, \mathbf{s}_{E}, \mathbf{r}_{E}\right)$ be a directed graph where $E^{0}$ is the set of vertices, $E^{1}$ the set of edges, and $\mathbf{s}_{E}, \mathbf{r}_{E}: E^{1} \rightarrow E^{0}$ are the source and the range maps. Since we consider just directed graphs, we refer to them simply as graphs. Also, if it is clear from the context, we write $\mathbf{s}_{E}$ and $\mathbf{r}_{E}$ shorter as $\mathbf{s}$ and r. A path $p$ in $E$ is a finite sequence of edges $p=e_{1} \ldots e_{n}$ such that $\mathbf{r}\left(e_{i}\right)=\mathbf{s}\left(e_{i+1}\right)$ for $i=1, \ldots, n-1$. Such path $p$ has length $n$ and we write $|p|=n$. The maps $\mathbf{s}$ and $\mathbf{r}$ extend to paths by $\mathbf{s}(p)=\mathbf{s}\left(e_{1}\right)$ and $\mathbf{r}(p)=\mathbf{r}\left(e_{n}\right)$. We consider vertices as paths of length zero. A path $p=e_{1} \ldots e_{n}$ is said to be closed if $\mathbf{s}(p)=\mathbf{r}(p)$. A closed path is said to be a cycle if $\mathbf{s}\left(e_{i}\right) \neq \mathbf{s}\left(e_{j}\right)$ for every $i \neq j$. A graph $E$ is said to be no-exit if $\mathbf{s}^{-1}(v)$ has just one element for every vertex $v$ of every cycle.

A vertex $v$ is said to be regular if the set $\mathbf{s}^{-1}(v)$ is nonempty and finite, $v$ is called a sink if $\mathbf{s}^{-1}(v)$ is empty, and $v$ is called an infinite emitter if $\mathbf{s}^{-1}(v)$ is infinite. A graph $E$ is row-finite if sinks are the only vertices that are not regular, finite if it is row-finite and $E^{0}$ is finite (in which case $E^{1}$ is necessarily finite as well), and countable if both $E^{0}$ and $E^{1}$ are countable.

For a graph $E$, consider the extended graph of $E$ to be the graph with the same vertices and with edges $E^{1} \cup\left\{e^{*} \mid e \in E^{1}\right\}$ where the range and source relations are the same as in $E$ for $e \in E^{1}$ and $\mathbf{s}\left(e^{*}\right)=\mathbf{r}(e)$ and $\mathbf{r}\left(e^{*}\right)=\mathbf{s}(e)$ for the added edges. Extend the map * to all the paths by defining $v^{*}=v$ for all vertices $v$ and $\left(e_{1} \ldots e_{n}\right)^{*}=e_{n}^{*} \ldots e_{1}^{*}$ for all paths $p=e_{1} \ldots e_{n}$. If $p$ is a path, we refer to elements of the form $p^{*}$ as ghost paths. Extend also the maps $\mathbf{s}$ and $\mathbf{r}$ to ghost paths by $\mathbf{s}\left(p^{*}\right)=\mathbf{r}(p)$ and $\mathbf{r}\left(p^{*}\right)=\mathbf{s}(p)$.

In the rest of the paper, $E$ denotes a graph and $K$ a field. The Cohn path algebra $C_{K}(E)$ of $E$ over $K$ is the free $K$-algebra generated by $E^{0} \cup E^{1} \cup\left\{e^{*} \mid e \in E^{1}\right\}$ subject to the following relations for all vertices $v, w$ and edges $e, f$.

(V) $v w=\delta_{v, w} v$

(E1) $\mathbf{s}(e) e=e \mathbf{r}(e)=e$,

(E2) $\mathbf{r}(e) e^{*}=e^{*} \mathbf{s}(e)=e^{*}$,

$(\mathrm{CK} 1) e^{*} f=\delta_{e, f} \mathbf{r}(e)$. 
The four axioms above imply that every element of $C_{K}(E)$ can be represented as a sum of the form $\sum_{i=1}^{n} a_{i} p_{i} q_{i}^{*}$ for some $n$, paths $p_{i}$ and $q_{i}$, and elements $a_{i} \in K$, for $i=1, \ldots, n$. We use $G_{E}$ to denote the set of all elements of the form $p q^{*}$ where $p$ and $q$ are paths with $\mathbf{r}(p)=\mathbf{r}(q)$.

If the underlying field $K$ has an involution $*$ (and there is always at least one such involution, the identity), the involution $*$ from $K$ extends to an involution of $C_{K}(E)$ by $\left(\sum_{i=1}^{n} a_{i} p_{i} q_{i}^{*}\right)^{*}=\sum_{i=1}^{n} a_{i}^{*} q_{i} p_{i}^{*}$ making $C_{K}(E)$ a $*$-algebra.

The Leavitt path algebra $L_{K}(E)$ of $E$ over $K$ is the free $K$-algebra generated by $E^{0} \cup E^{1} \cup$ $\left\{e^{*} \mid e \in E^{1}\right\}$ subject to relations (V), (E1), (E2), (CK1) and

(CK2) $v=\sum_{e \in \mathbf{s}^{-1}(v)} e e^{*}$ for every regular vertex $v$.

The Leavitt path algebra $L_{K}(E)$ can also be defined as the quotient $C_{K}(E) / N$ where $N$ is the ideal of the Cohn algebra $C_{K}(E)$ generated by all elements of the form $v-\sum_{e \in \mathbf{s}^{-1}(v)} e e^{*}$ where $v$ is a regular vertex. The algebra $L_{K}(E)$ is an involutive algebra with the involution inherited from $C_{K}(E)$. In some early works on Leavitt path algebras, the field $K$ was assumed to have the identity involution in all cases except when $K=\mathbb{C}$ in which case the involution was assumed to be the complex-conjugate involution. We stress the advantage of considering the base field $K$ as an involutive field with any involution and defining the involution on $L_{K}(E)$ by

$$
\left(\sum_{i=1}^{n} a_{i} p_{i} q_{i}^{*}\right)^{*}=\sum_{i=1}^{n} a_{i}^{*} q_{i} p_{i}^{*}
$$

for paths $p_{i}, q_{i}$ and $a_{i} \in K, i=1, \ldots, n$. This approach unifies the treatment of different involutive fields and integrates consideration of both the identity and the complex-conjugate involution on $\mathbb{C}$.

The underlying field $K$ does not play a role when characterizing many algebraic properties of Leavitt path algebras as shown in numerous papers. In fact, it has been hypothesized that two Leavitt path algebras isomorphic over one field are isomorphic over any other field. While this issue is still not settled, we point out that the presence of an involution definitely brings the underlying field into focus and makes properties of the field $K$ relevant for the "involution sensitive" ring-theoretic properties of $L_{K}(E)$. This is apparent in [5, Theorem 3.3] for example. We point out that Theorems 3.5 and 3.6 have the same sensitivity to involution on $K$. Namely, the assumptions of Theorems 3.5 and 3.6 imply that the field $K$ is positive definite as the next proposition shows. In this case, [5, Proposition 2.4] shows that $L_{K}(E)$ is positive definite for some (equivalently any) graph $E$.

Proposition 2.2. Let $R$ be an involutive $K$-algebra and $t: L_{K}(E) \rightarrow R$ a K-linear map on a Leavitt path algebra $L_{K}(E)$. If $R$ is positive definite and $t$ is faithful, then $K$ is positive definite thus $L_{K}(E)$ is positive definite as well.

Proof. If $R$ is positive definite and $t$ is faithful, then $t$ satisfies condition (2) of Lemma 2.1 by that Lemma. To show that $K$ is positive definite, note first that $K$ is proper since $K$ is a field: if $a \neq 0$ for $a \in K$ then $a^{*} \neq 0$ and so $a a^{*} \neq 0$ as well. Now let us assume that $\sum_{i=1}^{n} a_{i} a_{i}^{*}=0$ for some $a_{1}, \ldots, a_{n} \in K$. Then for any vertex $v$ we have that $0=\sum_{i=1}^{n} a_{i} a_{i}^{*} v=$ $\sum_{i=1}^{n}\left(a_{i} v\right)\left(a_{i} v\right)^{*}$ and so $t\left(\sum_{i=1}^{n}\left(a_{i} v\right)\left(a_{i} v\right)^{*}\right)=0$. Since condition (2) of Lemma 2.1 holds, this implies that $0=\left(a_{i} v\right)\left(a_{i} v\right)^{*}=a_{i} a_{i}^{*} v$ for each $i$. Assuming that $a_{i} a_{i}^{*} \neq 0$ we would have $v=0$ which is a contradiction. Thus $a_{i} a_{i}^{*}=0$ and so $a_{i}=0$ since $K$ is proper. Thus $K$ is positive definite. In this case, $L_{K}(E)$ is positive definite as well by [5, Proposition 2.4]. 
If a trace $t: L_{K}(E) \rightarrow R$ is positive for some $*$-ring $R$, then the trace values of vertices are positive elements of $R$. Let us denote this condition by $(\mathrm{P} 0)$.

$(\mathrm{P} 0) t(v) \geq 0$ for all vertices $v$.

[12, Proposition 29] lists more necessary conditions for a trace $t$ to be positive:

(P1) $t(v) \geq t(w)$ for all vertices $v$ and $w$, such that there is a path $p$ with $\mathbf{s}(p)=v$ and $\mathbf{r}(p)=w$.

(P2) $t(v) \geq \sum_{i=1}^{n} t\left(\mathbf{r}\left(e_{i}\right)\right)$ for all vertices $v$ and distinct edges $e_{1}, \ldots, e_{n}$ with $v$ as the source.

We show that (P2) implies (P1) and we combine conditions (P0) and (P2) into a single condition.

Lemma 2.3. If $R$ is a *-ring and $t$ is an $R$-valued trace on $L_{K}(E)$, conditions (P0) and $(P 2)$ are equivalent to condition $(P)$ below and imply (P1).

(P) $\quad t\left(v-\sum_{e \in I} \mathbf{r}(e)\right) \geq 0$ for all vertices $v$ and finite subsets $I$ of $\mathbf{s}^{-1}(v)$.

Proof. Condition (P) is condition (P0) in case when the set $I$ is empty. If $I$ is nonempty, conditions $(\mathrm{P} 2)$ and $(\mathrm{P})$ are equivalent since $t\left(v-\sum_{e \in I} \mathbf{r}(e)\right)=t(v)-\sum_{e \in I} t(\mathbf{r}(e))$.

Let us show that (P2) implies (P1). Let $v, w$ and $p$ be as in $(\mathrm{P} 1)$. We prove the claim by induction on the length of $p$. If $|p|=0, v=w$ and (P1) clearly holds. Assuming (P1) for paths of length $n$, let us prove $(\mathrm{P} 1)$ for a path $p=e p_{1}$ where $\mathbf{s}(e)=v, \mathbf{r}(e)=\mathbf{s}\left(p_{1}\right)$, $\mathbf{r}\left(p_{1}\right)=w$, and $\left|p_{1}\right|=n$. Indeed, $t(v) \geq t(\mathbf{r}(e))$ by $(\mathrm{P} 2)$ and $t(\mathbf{r}(e)) \geq t(w)$ by the induction hypothesis. Thus, $t(v) \geq t(w)$.

Condition (F) below is clearly necessary for a trace $t: L_{K}(E) \rightarrow R$ to be faithful for some *-ring $R$.

(F) $t(v)>0$ for all vertices $v$.

Neither $(\mathrm{P})$ is sufficient for positivity nor $(\mathrm{P})$ and $(\mathrm{F})$ are sufficient for faithfulness of a trace on a Leavitt path algebra as it was observed in [12, Example 30]. In this example, $\mathbb{C}\left[x, x^{-1}\right]$ was considered as the Leavitt path algebra of the single-vertex single-edge graph over $\mathbb{C}$. With the complex-conjugate involution on $\mathbb{C}$, the trace defined by $t\left(x^{n}\right)=i^{n}$ for $n \geq 0$ and $t\left(x^{n}\right)=i^{-n}$ for $n<0$ is such that $(\mathrm{P})$ and $(\mathrm{F})$ hold. However, by considering the trace of the positive element $(1+x)\left(1+x^{-1}\right)$ one can see that $t$ is not positive (thus also not faithful).

The fact that $(\mathrm{P})$ is not sufficient for positivity and $(\mathrm{P})$ and $(\mathrm{F})$ are not sufficient for faithfulness of a trace should not be surprising since traces are rather general classes of maps. We show that this drawback is not present for a certain class of well-behaved traces. We refer to such traces as canonical traces. This terminology will be justified in Proposition 2.7. We define a canonical trace using the following proposition.

Proposition 2.4. Any map $t$ on $G_{E}=\left\{p q^{*} \mid p, q\right.$ paths with $\left.\mathbf{r}(p)=\mathbf{r}(q)\right\}$ such that

$$
t\left(p q^{*}\right)=\delta_{p, q} t(\mathbf{r}(p))
$$

uniquely extends to a $K$-linear trace on $L_{K}(E)$.

If $t$ is a trace on $L_{K}(E)$, the following conditions are equivalent.

(1) $t\left(p q^{*}\right)=\delta_{p, q} t(\mathbf{r}(p))$ for all paths $p$ and $q$.

(2) $t\left(p q^{*}\right)=0$ for all paths $p$ and $q$ of non-equal length. 
Conditions (1) and (2) imply condition (3) below. If $K$ has characteristic zero, then the conditions (1) and (2) are equivalent to (3).

(3) $t\left(p q^{*}\right)=k^{|p|-|q|} t\left(p q^{*}\right)$ for any nonzero $k \in K$.

Proof. To show the first sentence, consider [12, Proposition 19] proving that any map $\delta$ on $G_{E} \cup\{0\}$ which preserves zero is central if and only if the following three conditions hold:

(i) If $\delta(x) \neq 0$ for some $x \in G_{E}$, then either $x=p q p^{*}$ or $x=p q^{*} p^{*}$ for some path $p$ and some closed path $q$.

(ii) $\delta\left(p q p^{*}\right)=\delta(q)$ and $\delta\left(p q^{*} p^{*}\right)=\delta\left(q^{*}\right)$ for any path $p$ and any closed path $q$.

(iii) $\delta(p)=\delta(q)$ and $\delta\left(p^{*}\right)=\delta\left(q^{*}\right)$ for any two closed paths $p$ and $q$ such that $x y=p$ and $y x=q$ for some paths $x$ and $y$.

It is easy to check that the map $t$ as in the first sentence of the proposition satisfies these three conditions. Thus $t$ is a central map on $G_{E}$. Since every element of $L_{K}(E)$ is a $K$-linear combination of elements from $G_{E}$, the map $t$ extends to a $K$-linear trace of $L_{K}(E)$. This extension is unique since if two $K$-linear maps agree on $G_{E}$ then they are equal on $L_{K}(E)$.

If $t$ is a trace on $L_{K}(E)$, let us show the equivalence of conditions (1) and (2). (1) clearly implies (2). Since $t\left(p p^{*}\right)=t\left(p^{*} p\right)=t(\mathbf{r}(p))$, to show the converse it is sufficient to show that $t\left(p q^{*}\right) \neq 0$ and $|p|=|q|$ imply $p=q$. Let us use induction on the length $|p|=|q|$. If $p$ and $q$ are vertices, the claim clearly holds by axiom $(\mathrm{V})$.

Assume that the claim holds for paths $p$ and $q$ with $|p|=|q|=n$ and let us prove the claim for paths $p=e p_{1}$ and $q=f q_{1}$ where $e$ and $f$ are edges and $p_{1}$ and $q_{1}$ paths with $\left|p_{1}\right|=\left|q_{1}\right|=n, \mathbf{r}(e)=\mathbf{s}\left(p_{1}\right)$ and $\mathbf{r}(f)=\mathbf{s}\left(q_{1}\right)$. Then $0 \neq t\left(p q^{*}\right)=t\left(e p_{1} q_{1}^{*} f^{*}\right)=t\left(f^{*} e p_{1} q_{1}^{*}\right)$ implies that $f^{*} e p_{1} q_{1}^{*} \neq 0$ and so $f^{*} e \neq 0$ thus $e=f$. Then we can use the induction hypothesis for $t\left(p_{1} q_{1}^{*}\right)=t\left(e^{*} e p_{1} q_{1}^{*}\right)=t\left(f^{*} e p_{1} q_{1}^{*}\right)=t\left(e p_{1} q_{1}^{*} f^{*}\right) \neq 0$ to obtain that $p_{1}=q_{1}$. Thus $p=e p_{1}=e q_{1}=q$.

(1) implies (3). Indeed, if $k \in K$ is nonzero and $p \neq q$, then $t\left(p q^{*}\right)=z^{|p|-|q|} t\left(p q^{*}\right)$ trivially holds since both sides are zero by (1). If $p=q$, then $t\left(p p^{*}\right)=z^{|p|-|p|} t\left(p p^{*}\right)$ also holds.

Now let us assume that (3) holds and that char $K=0$ and let us show (2). Assume that $|p| \neq|q|$. Then

$$
t\left(p q^{*}\right)=k^{|p|-|q|} t\left(p q^{*}\right) \text { implies }\left(1-k^{|p|-|q|}\right) t\left(p q^{*}\right)=0 \text { for every } 0 \neq k \in K .
$$

Since $\operatorname{char} K=0$, we can find a nonzero element $k$ in $K$ that is not a $(|p|-|q|)$-th root of the identity in $K$ in case $|p|>|q|$. If $|q|>|p|$, consider $k^{-1}$ for $k$ that is not a $(|q|-|p|)$-th root of the identity. In both cases, $1-k^{|p|-|q|} \neq 0$ and so $t\left(p q^{*}\right)=0$.

Condition (3) from Proposition 2.4 is the algebraic version of the definition of a gauge invariant trace on a graph $C^{*}$-algebra. Namely, the gauge action on a graph $C^{*}$-algebra given as in [2, Definition 2.13] generalizes to Leavitt path algebras as follows.

The gauge action on $L_{K}(E)$ is a group homomorphism $\lambda: K \backslash\{0\} \rightarrow \operatorname{Aut}\left(L_{K}(E)\right)$ such that for any $0 \neq k \in K, \lambda(k)(v)=v$ for all vertices $v, \lambda(k)(e)=k e$ and $\lambda(k)\left(e^{*}\right)=\frac{1}{k} e^{*}$ for all edges $e$. It is easy to see that in this case

$$
\lambda(k)\left(p q^{*}\right)=k^{|p|-|q|} p q^{*}
$$

for any paths $p$ and $q$. This fact and Proposition 2.4 motivate the following definition.

Definition 2.5. If $t$ is a trace on $L_{K}(E)$ and $p$ and $q$ paths, then 
(1) $t$ is gauge invariant if

$$
t\left(p q^{*}\right)=k^{|p|-|q|} t\left(p q^{*}\right) \quad \text { for any nonzero } k \in K .
$$

(2) $t$ is canonical if

$$
t\left(p q^{*}\right)=\delta_{|p|,|q|} t\left(p q^{*}\right)=\delta_{p, q} t\left(p q^{*}\right)=\delta_{p, q} t(\mathbf{r}(p)) .
$$

The equalities in (2) follow from Proposition 2.4. Also by Proposition 2.4, every canonical trace is gauge invariant and the converse is also true if $K$ has characteristic zero.

We justify the use of the term canonical by Proposition 2.7 which shows that every canonical trace is uniquely determined by its value on vertices. Note that some maps on vertices cannot be extended to traces because their values may be such that axiom (CK2) is violated. For example, consider the graph $E$ below and the $\mathbb{C}$-valued map which maps $u$ and $w$ to 1 and $v$ to 3 .

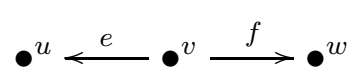

This map satisfies conditions $(\mathrm{P})$ and $(\mathrm{F})$ but it cannot be extended to a $\mathbb{C}$-valued trace $t$ on the Leavitt path algebra $L_{\mathbb{C}}(E)$ since $3=t(v)=t\left(e e^{*}+f f^{*}\right)=t\left(e^{*} e\right)+t\left(f^{*} f\right)=$ $t(u)+t(w)=2$ in that case.

This example illustrates that the values on vertices have to agree with (CK2). This fact was noticed by Tomforde in [16] and later also utilized in [10] and [14]. Tomforde considers maps $\delta$ on vertices with values in $(0, \infty)$ which satisfy the following two conditions and calls them graph traces on $E$.

(1) For all regular vertices $v$ we have $\delta(v)=\sum_{e \in \mathbf{s}^{-1}(v)} \delta(\mathbf{r}(e))$.

(2) For all infinite emitters $v$ and every $e_{1}, \ldots, e_{n} \in \mathbf{s}^{-1}(v), \delta(v) \geq \sum_{i=1}^{n} \delta(\mathbf{r}(e))$.

Since condition (2) follows from condition (P), we define a graph trace by condition (1) only. We also allow the values of a graph trace to be in any involutive $K$-algebra $R$ not necessarily real interval $(0, \infty)$.

Definition 2.6. If $R$ is a ring, a map $\delta: E^{0} \rightarrow R$ is a graph trace on $E$ if

$(\mathrm{CK} 2)_{\delta} \quad \delta(v)=\sum_{e \in \mathbf{s}^{-1}(v)} \delta(\mathbf{r}(e))$ for all regular vertices $v$.

If $R$ is a *-ring, the graph trace $\delta$ is positive if

$(\mathrm{P})_{\delta} \quad \delta(v) \geq \sum_{e \in I} \delta(\mathbf{r}(e)) \quad$ for all vertices $v$ and finite subsets $I$ of $\mathbf{s}^{-1}(v)$

where $\sum_{e \in \emptyset} \delta(\mathbf{r}(e))$ is defined to be 0 .

A positive graph trace $\delta$ is faithful if

$(\mathrm{F})_{\delta} \quad \delta(v)>0$ for all vertices $v$.

Proposition 2.7. Let $R$ be a K-algebra. There is a bijective correspondence $\tau$ between

(1) $R$-valued graph traces on a graph $E$ and

(2) canonical, $K$-linear, $R$-valued traces on $L_{K}(E)$.

Proof. Let $\delta$ be a graph trace on $E$. Define the map $t_{\delta}$ on $G_{E}$ by $t_{\delta}\left(p q^{*}\right)=\delta_{p, q} \delta(\mathbf{r}(p))$ and let $t_{\delta}(0)=0$. By [12, Theorem 28], $t_{\delta}$ is central and extends to a $K$-linear, $R$-valued trace $t_{\delta}$ on $L_{K}(E)$. The trace $t_{\delta}$ is canonical by construction and its restriction to vertices is $\delta$. 
Conversely, if $t$ is a canonical, $K$-linear, $R$-valued trace on $L_{K}(E)$, then the restriction $\delta$ of $t$ to $E^{0}$ is a graph trace by axioms (CK1), (CK2) and the fact that $t$ is central:

$$
\delta(v)=t\left(\sum_{e \in \mathbf{s}^{-1}(v)} e e^{*}\right)=\sum_{e \in \mathbf{s}^{-1}(v)} t\left(e e^{*}\right)=\sum_{e \in \mathbf{s}^{-1}(v)} t\left(e^{*} e\right)=\sum_{e \in \mathbf{s}^{-1}(v)} t(\mathbf{r}(e))=\sum_{e \in \mathbf{s}^{-1}(v)} \delta(\mathbf{r}(e))
$$

for any regular vertex $v$. Then $t\left(p q^{*}\right)=\delta_{p, q} \delta(\mathbf{r}(p))=t_{\delta}\left(p q^{*}\right)$ for all paths $p$ and $q$ since $t$ is canonical. Thus $t=t_{\delta}$ on $G_{E}$ and, consequently, $t=t_{\delta}$ on $L_{K}(E)$.

\section{Characterizations of positive and faithful Canonical traces}

By Proposition 2.7, a canonical, $K$-linear trace on $L_{K}(E)$ can be seen as a well-behaved representative of all traces on $L_{K}(E)$ with the same values on vertices. In this section, we prove similar characterizations of canonical, $K$-linear traces which are positive (Theorem 3.4) and which are faithful (Theorem 3.5). As a consequence, there is a bijective correspondence between positive, canonical, $K$-linear traces and positive graph traces and a bijective correspondence between faithful, canonical, $K$-linear traces with values in a positive definite algebra and faithful graph traces (Theorem 3.6). We start by several lemmas.

Lemma 3.1. If $t$ is a canonical trace on $L_{K}(E)$ and $p, q, r, s$ any paths then $t\left(p q^{*} r s^{*}\right) \neq 0$ implies that either

$$
\text { Case 1. } s=p u \text { and } r=q u \text {, or } \quad \text { Case 2. } p=s u \text { and } q=r u
$$

for some path $u$. In both cases $t\left(p q^{*} r s^{*}\right)=t\left(u u^{*}\right)=t(\mathbf{r}(u))$.

Proof. If $t\left(p q^{*} r s^{*}\right) \neq 0$ then $t\left(s^{*} p q^{*} r\right) \neq 0$ so $s^{*} p q^{*} r \neq 0$. This implies that either $s=p u$ or $p=s u$ and $r=q v$ or $q=r v$ for some paths $u$ and $v$.

If $s=p u$ and $r=q v$ then $p q^{*} r s^{*}=p q^{*} q v u^{*} p^{*}=p v u^{*} p^{*} \neq 0$. So $0 \neq t\left(p v u^{*} p^{*}\right)=$ $t\left(p^{*} p v u^{*}\right)=t\left(v u^{*}\right)$ implies that $u=v$ since $t$ is canonical. If $p=s u$ and $q=r v$, we obtain $u=v$ similarly.

If $s=p u$ and $q=r v$, then $p q^{*} r s^{*}=p v^{*} r^{*} r u^{*} p^{*}=p v^{*} u^{*} p^{*} \neq 0$. So $0 \neq t\left(p v^{*} u^{*} p^{*}\right)=$ $t\left(p^{*} p v^{*} u^{*}\right)=t\left(v^{*} u^{*}\right)$ implies that $v^{*} u^{*}$ is a vertex (necessarily $\mathbf{r}(q)=\mathbf{r}(s)$ in this case.) Thus $s=p$ and $q=r$. Hence this case falls under the previous case with $u=v=\mathbf{r}(p)=\mathbf{r}(q)$. We reach a similar conclusion if $p=s u$ and $r=q v$.

Thus, we have that either (Case 1) $s=p u$ and $r=q u$ so $r s^{*}=q u u^{*} p^{*}$ in which case $t\left(p q^{*} r s^{*}\right)=t\left(p u u^{*} p^{*}\right)=t\left(u u^{*}\right)=t(\mathbf{r}(u))$, or (Case 2) $p=s u$ and $q=r u$ so $p q^{*}=s u u^{*} r^{*}$ in which case $t\left(p q^{*} r s^{*}\right)=t\left(s u u^{*} s^{*}\right)=t\left(u u^{*}\right)=t(\mathbf{r}(u))$.

Let us define a partial order $\preceq$ on the set $G_{E}=\left\{p q^{*} \mid p\right.$ and $q$ paths with $\left.\mathbf{r}(p)=\mathbf{r}(q)\right\}$ by

$$
p u u^{*} q^{*} \preceq p q^{*} \text { for any path } u
$$

in which case we say that $p u u^{*} q^{*}$ is reducible to $p q^{*}$.

We say that $p q^{*}$ is irreducible if $p q^{*} \preceq r s^{*}$ implies that $p=r$ and $q=s$. If $p q^{*}$ is irreducible, $p=r u$ and $q=s u$ only for paths $u$ of length zero.

We refer to the $G_{E}$ elements of the form $p u_{1} u_{1}^{*} q^{*}$ and $p u_{2} u_{2}^{*} q^{*}$ as comparable and we write

$$
p u_{1} u_{1}^{*} q^{*} \sim p u_{2} u_{2}^{*} q^{*}
$$

in this case. Such two comparable elements can both be reduced to $p q^{*}$. The following lemma establishes that comparability is an equivalence relation and that elements of a finite set of comparable elements can be reduced to the same irreducible element. 
Lemma 3.2. (1) Every element of $G_{E}$ can be reduced to a unique irreducible element.

(2) Two elements of $G_{E}$ are comparable if and only if they can be reduced to the same irreducible element. Such irreducible element is unique.

(3) Relation $\sim$ is an equivalence relation on $G_{E}$ and elements from the same equivalence class reduce to the same irreducible element, necessarily unique.

(4) If $t\left(p q^{*}\left(r s^{*}\right)^{*}\right) \neq 0$ for some canonical trace $t$ on $L_{K}(E)$ and some $p q^{*}, r s^{*} \in G_{E}$, then $p q^{*}$ and $r s^{*}$ are comparable.

Proof. (1) Consider $p q^{*} \in G_{E}$. If $p q^{*}$ is irreducible, we are done. If not, $p=r u$ and $q=s u$ for some path $u$ of nonzero length and $r s^{*} \in G_{E}$. If $r s^{*}$ is irreducible, we are done. If not, repeat the argument for $r s^{*}$. Since we are either shortening the length of paths in each step or we end up with an irreducible element, the process ends after finitely many steps and we arrive to an irreducible upper bound of $p q^{*}$.

To show uniqueness, assume that there are irreducible elements $r_{1} s_{1}^{*}$ and $r_{2} s_{2}^{*}$ of $G_{E}$ and paths $t_{1}, t_{2}$ such that $p q^{*}=r_{1} t_{1} t_{1}^{*} s_{1}^{*}$ and $p q^{*}=r_{2} t_{2} t_{2}^{*} s_{2}^{*}$. These relations imply that $p=r_{1} t_{1}=r_{2} t_{2}, q=s_{1} t_{1}=s_{2} t_{2}$. Thus we have that either $r_{1}$ is a prefix of $r_{2}$ or vice versa and that $s_{1}$ is a prefix of $s_{2}$ or vice versa. In any of these cases, we claim that $r_{1}=r_{2}$ and $s_{1}=s_{2}$.

If $r_{1}$ is a prefix of $r_{2}$ and $s_{1}$ is a prefix of $s_{2}$, then $r_{2}=r_{1} u_{1}$ and $s_{2}=s_{1} u_{2}$ for some paths $u_{1}$ and $u_{2}$. In this case, $r_{1} t_{1}=r_{1} u_{1} t_{2}$ and $s_{1} t_{1}=s_{1} u_{2} t_{2}$ and so $t_{1}=u_{1} t_{2}=u_{2} t_{2}$ which implies $u_{1}=u_{2}$. Then we have that $r_{2} s_{2}^{*}=r_{1} u_{1} u_{1}^{*} s_{1}^{*} \preceq r_{1} s_{1}^{*}$ and so $u_{1}$ has to be a path of length zero by irreducibility of $r_{2} s_{2}^{*}$. Thus $r_{2}=r_{1} u_{1}=r_{1}$ and $s_{2}=s_{1} u_{1}=s_{1}$. The case when $r_{2}$ is a prefix of $r_{1}$ and $s_{2}$ is a prefix of $s_{1}$ is handled similarly.

If $r_{1}$ is a prefix of $r_{2}$ and $s_{2}$ is a prefix of $s_{1}$, then $r_{2}=r_{1} u_{1}$ and $s_{1}=s_{2} u_{2}$ for some paths $u_{1}$ and $u_{2}$. In this case, $r_{1} t_{1}=r_{1} u_{1} t_{2}$ and $s_{2} u_{2} t_{1}=s_{2} t_{2}$ and so $t_{1}=u_{1} t_{2}$ and $u_{2} t_{1}=t_{2}$ which implies $u_{1} u_{2} t_{1}=t_{1}$ and $u_{2} u_{1} t_{2}=t_{2}$. This implies that $u_{1} u_{2}$ and $u_{2} u_{1}$ are paths of length zero and so $u_{1}=u_{2}$ is a vertex. Thus, $r_{2}=r_{1} u_{1}=r_{1}$ and $s_{1}=s_{2} u_{2}=s_{2} u_{1}=s_{2}$. The case when $r_{2}$ is a prefix of $r_{1}$ and $s_{1}$ is a prefix of $s_{2}$ is handled similarly. Since $r_{1}=r_{2}$ and $s_{1}=s_{2}$ in any case, $p q^{*}$ reduces to a unique irreducible element.

(2) Let $p_{1} q_{1}^{*}$ and $p_{2} q_{2}^{*}$ be comparable elements of $G_{E}$. We claim that they reduce to the same irreducible element. Since $p_{1} q_{1}^{*}$ and $p_{2} q_{2}^{*}$ are comparable, $p_{1} q_{1}^{*}=p u_{1} u_{1}^{*} q^{*}$ and $p_{2} q_{2}^{*}=p u_{2} u_{2}^{*} q^{*}$ for some $p q^{*} \in G_{E}$ and paths $u_{1}, u_{2}$. Let $r s^{*}$ be the irreducible element to which $p q^{*}$ reduces. Thus $p q^{*}=r u u^{*} s^{*}$ for some path $u$. Thus $p_{1} q_{1}^{*}=r u u_{1} u_{1}^{*} u^{*} s^{*}$ and $p_{2} q_{2}^{*}=r u u_{2} u_{2}^{*} u^{*} s^{*}$ and so $p_{1} q_{1}^{*}$ and $p_{2} q_{2}^{*}$ reduce to irreducible $r s^{*}$. The uniqueness of $r s^{*}$ follows from part (1).

The converse follows by the definition of comparability.

(3) Relation $\sim$ is clearly reflexive and symmetric. To show transitivity, let $p_{i} q_{i}^{*} \in G_{E}, i=$ $1,2,3, p_{1} q_{1}^{*} \sim p_{2} q_{2}^{*}$, and $p_{2} q_{2}^{*} \sim p_{3} q_{3}^{*}$. By part (2), there are irreducible elements $p q^{*}$ and $r s^{*}$ of $G_{E}$ such that $p_{1} q_{1}^{*}$ and $p_{2} q_{2}^{*}$ reduce to $p q^{*}$ and $p_{2} q_{2}^{*}$ and $p_{3} q_{3}^{*}$ reduce to $r s^{*}$. Then $p_{2} q_{2}^{*}$ reduces to both $p q^{*}$ and $r s^{*}$. By (1), $p=r$ and $q=s$ and all three elements reduce to $p q^{*}$. Thus, $p_{1} q_{1}^{*} \sim p_{3} q_{3}^{*}$ by part (2).

The second part of the claim follows from the transitivity of $\sim$ and parts (1) and (2).

(4) If $t\left(p q^{*}\left(r s^{*}\right)^{*}\right)=t\left(p q^{*} s r^{*}\right) \neq 0$ for some canonical trace $t$ on $L_{K}(E)$ and some $p q^{*}, r s^{*} \in$ $G_{E}$, then either $r=p u$ and $s=q u$ or $p=r u$ and $q=s u$ for some path $u$ by Lemma 3.1. In the first case $r s^{*}=p u u^{*} q^{*}$ and in the second case $p q^{*}=r u u^{*} s^{*}$. In both cases $p q^{*}$ and $r s^{*}$ are comparable.

The following lemma is the last one we need for the proof of Theorem 3.4 . 
Lemma 3.3. Let $t$ be a canonical, $K$-linear trace on $L_{K}(E)$.

(1) If $a_{i} \in K$, and $r_{i} r_{i}^{*}, p r_{i} r_{i}^{*} q^{*} \in G_{E}$ for $i=1, \ldots, m$,

$$
x=\sum_{i=1}^{m} a_{i} p r_{i} r_{i}^{*} q^{*} \quad \text { and } \quad y=\sum_{i=1}^{m} a_{i} r_{i} r_{i}^{*}
$$

then

$$
t\left(x x^{*}\right)=t\left(y y^{*}\right)
$$

(2) If $t$ satisfies condition (P) from Lemma 2.3,

$$
x=\sum_{i=1}^{m} \sum_{j=1}^{m_{i}} a_{i j} e_{i} r_{i j} r_{i j}^{*} e_{i}^{*}+a v \quad \text { and } \quad y=\sum_{i=1}^{m} \sum_{j=1}^{m_{i}} a_{i j} e_{i} r_{i j} r_{i j}^{*} e_{i}^{*}+a \sum_{i=1}^{m} e_{i} e_{i}^{*}
$$

where $v$ is a vertex of $E,\left\{e_{1}, \ldots, e_{m}\right\} \subseteq \mathbf{s}^{-1}(v), e_{i} \neq e_{j}$ for $i \neq j, a, a_{i j} \in K$, $e_{i} r_{i j} r_{i j}^{*} e_{i}^{*} \in G_{E}$ for $i=1, \ldots, m$ and $j=1, \ldots, m_{i}$, then

$$
t\left(x x^{*}\right) \geq t\left(y y^{*}\right) \text {. }
$$

Proof. To prove (1), note that

$$
x x^{*}=\sum_{i=1}^{m} \sum_{j=1}^{m} a_{i} a_{j}^{*} p r_{i} r_{i}^{*} q^{*} q r_{j} r_{j}^{*} p^{*}=\sum_{i=1}^{m} \sum_{j=1}^{m} a_{i} a_{j}^{*} p r_{i} r_{i}^{*} r_{j} r_{j}^{*} p^{*} \text { and } y y^{*}=\sum_{i=1}^{m} \sum_{j=1}^{m} a_{i} a_{j}^{*} r_{i} r_{i}^{*} r_{j} r_{j}^{*} \text {. }
$$

Thus,

$$
t\left(x x^{*}\right)=\sum_{i=1}^{m} \sum_{j=1}^{m} a_{i} a_{j}^{*} t\left(p r_{i} r_{i}^{*} r_{j} r_{j}^{*} p^{*}\right)=\sum_{i=1}^{m} \sum_{j=1}^{m} a_{i} a_{j}^{*} t\left(p^{*} p r_{i} r_{i}^{*} r_{j} r_{j}^{*}\right)=\sum_{i=1}^{m} \sum_{j=1}^{m} a_{i} a_{j}^{*} t\left(r_{i} r_{i}^{*} r_{j} r_{j}^{*}\right)
$$

This last expression is equal to $t\left(y y^{*}\right)$.

To prove (2), compute that

$$
x x^{*}=\sum_{i=1}^{m} \sum_{j=1}^{m_{i}} \sum_{k=1}^{m_{i}} a_{i j} a_{i k}^{*} e_{i} r_{i j} r_{i j}^{*} r_{i k} r_{i k}^{*} e_{i}^{*}+\sum_{i=1}^{m} \sum_{j=1}^{m_{i}}\left(a_{i j} a^{*}+a a_{i j}^{*}\right) e_{i} r_{i j} r_{i j}^{*} e_{i}^{*}+a a^{*} v
$$

by using the fact that $e_{i} \neq e_{j}$ for $i \neq j$ and that $\mathbf{s}\left(e_{i}\right)=v$ for all $i, j=1, \ldots, m$. Similarly,

$$
y y^{*}=\sum_{i=1}^{m} \sum_{j=1}^{m_{i}} \sum_{k=1}^{m_{i}} a_{i j} a_{i k}^{*} e_{i} r_{i j} r_{i j}^{*} r_{i k} r_{i k}^{*} e_{i}^{*}+\sum_{i=1}^{m} \sum_{j=1}^{m_{i}}\left(a_{i j} a^{*}+a a_{i j}^{*}\right) e_{i} r_{i j} r_{i j}^{*} e_{i}^{*}+a a^{*} \sum_{i=1}^{m} e_{i} e_{i}^{*} .
$$

Since the underlined parts are equal,

$$
\begin{gathered}
t\left(x x^{*}-y y^{*}\right)=t\left(a a^{*} v-a a^{*} \sum_{i=1}^{m} e_{i} e_{i}^{*}\right)=a a^{*}\left(t(v)-\sum_{i=1}^{m} t\left(e_{i} e_{i}^{*}\right)\right)= \\
=a a^{*}\left(t(v)-\sum_{i=1}^{m} t\left(\mathbf{r}\left(e_{i}\right)\right)\right)=a a^{*} t\left(v-\sum_{i=1}^{m} \mathbf{r}\left(e_{i}\right)\right) \geq 0
\end{gathered}
$$

by $(\mathrm{P})$. Thus $t\left(x x^{*}\right) \geq t\left(y y^{*}\right)$.

We can now prove the main results of this section starting with the following.

Theorem 3.4. Let $t$ be a canonical, $K$-linear trace on $L_{K}(E)$. Then $t$ is positive if and only if 
(P) $\quad t\left(v-\sum_{e \in I} \mathbf{r}(e)\right) \geq 0$ for all vertices $v$ and finite subsets $I$ of $\mathbf{s}^{-1}(v)$.

Proof. If $t$ is positive, then condition (P) holds by [12, Proposition 29] and Lemma 2.3.

To prove the converse, it is sufficient to show that $t\left(x x^{*}\right) \geq 0$ for every $x \in L_{K}(E)$. The elements of $G_{E}$ generate $L_{K}(E)$ as a $K$-algebra so it is sufficient to assume that $x$ is a $K$-linear combination of $G_{E}$ elements. Such $x$ can be written as

$$
x=\sum_{i=1}^{n} x_{i}
$$

where the elements $x_{i}$ are $K$-linear combinations of comparable elements reducible to the same irreducible element $p_{i} q_{i}^{*} \in G_{E}$ and all irreducible elements $p_{i} q_{i}^{*}, p_{j} q_{j}^{*}$ are different, thus not comparable, for $i \neq j$. This representation of $x$ is possible by part (3) of Lemma 3.2, Since $t\left(x_{i} x_{j}^{*}\right)=0$ for $i \neq j$ by construction and part (4) of Lemma 3.2, $t\left(x x^{*}\right)=\sum_{i=1}^{n} t\left(x_{i} x_{i}^{*}\right)$.

Thus, it is sufficient to consider elements $x$ which are $K$-linear combinations of $G_{E}$ elements comparable to each other. Let $x$ be one such element. By part (3) of Lemma 3.2, there is an irreducible $G_{E}$ element $p q^{*}$ such that $x$ can be written as

$$
x=\sum_{j=1}^{l} a_{j} p r_{j} r_{j}^{*} q^{*}
$$

where $p r_{j} r_{j}^{*} q^{*} \in G_{E}$ and $a_{j} \in K$ for $j=1, \ldots, l$. By part (1) of Lemma 3.3, $t\left(x x^{*}\right)=t\left(y y^{*}\right)$ where $y=\sum_{i=j}^{l} a_{j} r_{j} r_{j}^{*}$. Note that all paths $r_{j}$ have the same source $\mathbf{r}(p)$ since $p r_{j} \neq 0$. Thus, it is sufficient to consider elements $x$ of the form

$$
x=\sum_{j=1}^{l} a_{j} r_{j} r_{j}^{*}, \quad \mathbf{s}\left(r_{i}\right)=\mathbf{s}\left(r_{j}\right) \text { for all } i, j=1, \ldots, l .
$$

Let $v$ denote the source of all $r_{j}, j=1, \ldots, l$. If several different paths $r_{j}$ have zero length, group them in a single term by writing $a v+b v$ as $(a+b) v$ for $a, b \in K$. Depending on the coefficient with $v$ being zero or nonzero, we have three possible cases.

Case 1 . None of the paths $r_{j}$ have zero length.

Case 2. Exactly one of the paths $r_{j}$ has zero length and $l=1$.

Case 3. Exactly one of the paths $r_{j}$ has zero length and $l>1$.

In case 1 , let $e_{i}, i=1, \ldots, m$ be the list of all edges that are the first in paths $r_{j}, j=1, \ldots, l$ without repetition. Let us denote every $r_{j}$ as $e_{i} r_{i k}$ for some paths $r_{i k}$ in which case we write $a_{j}$ as $a_{i k}$. Then we can write $x$ as

$$
x=\sum_{i=1}^{m} x_{i} \quad \text { for } \quad x_{i}=\sum_{k=1}^{m_{i}} a_{i k} e_{i} r_{i k} r_{i k}^{*} e_{i}^{*}
$$

and $l=\sum_{i=1}^{m} m_{i}$ necessarily. If $i \neq j, e_{i}^{*} e_{j}=0$ and so $x_{i} x_{j}^{*}=0$. Thus $t\left(x x^{*}\right)=\sum_{i=1}^{m} t\left(x_{i} x_{i}^{*}\right)$.

For every $x_{i}, i=1, \ldots m$, we can apply part (1) of Lemma 3.3 to obtain that $t\left(x_{i} x_{i}^{*}\right)=$ $t\left(z_{i} z_{i}^{*}\right)$ where

$$
z_{i}=\sum_{k=1}^{m_{i}} a_{i k} r_{i k} r_{i k}^{*}
$$

In case $2, l=1$ and $x$ has the form $x=a_{1} v$. Since $t(v)$ is positive by condition $(\mathrm{P})$, $t\left(x x^{*}\right)=a_{1} a_{1}^{*} t(v)$ is positive as well. 
In case 3 , we show that the consideration reduces to either case 1 or case 2 . Since there is one $r_{j}$ with zero length, we can assume it is $r_{l}$. Let $e_{i}, i=1, \ldots, m$ be the list of all edges that are the first in paths $r_{j}, j=1, \ldots, l-1$ without repetition. Let $r_{j}=e_{i} r_{i k}$ for some paths $r_{i k}$ and let us represent $a_{j}$ as $a_{i k}$ so that we can write $x$ as

$$
x=\sum_{i=1}^{m} \sum_{k=1}^{m_{i}} a_{i k} e_{i} r_{i k} r_{i k}^{*} e_{i}^{*}+a_{l} v .
$$

Here $l-1$ is necessarily equal to $\sum_{i=1}^{m} m_{i}$. In this case, $t\left(x x^{*}\right) \geq t\left(y y^{*}\right)$ where

$$
y=\sum_{i=1}^{m} \sum_{k=1}^{m_{i}} a_{i k} e_{i} r_{i k} r_{i k}^{*} e_{i}^{*}+a_{l} \sum_{i=1}^{m} e_{i} e_{i}^{*}
$$

by part (2) of Lemma 3.3. So, it is sufficient to show that the trace of $y y^{*}$ is positive. Since we can regroup the terms of $y$ so that

$$
y=\sum_{i=1}^{m} y_{i} \quad \text { for } \quad y_{i}=\sum_{k=1}^{m_{i}} a_{i k} e_{i} r_{i k} r_{i k}^{*} e_{i}^{*}+a_{l} e_{i} e_{i}^{*},
$$

the element $y$ falls under case 1 and is represented as in (44). In this case the elements $z_{i}=\sum_{k=1}^{m_{i}} a_{i k} r_{i k} r_{i k}^{*}+a_{l} \mathbf{r}\left(e_{i}\right)$ are as in (5) and $t\left(y_{i} y_{i}^{*}\right)=t\left(z_{i} z_{i}^{*}\right)$. Thus, we either reduce our consideration to $z_{i}$ as in (5) of case 1 or the elements $z_{i}$ already have the form as in case 2 .

All paths $r_{j}$ of nonzero length in formula (3) have strictly longer length than the paths $r_{i k}$ in (5) since $r_{j}=e_{i} r_{i k}$. The expression in formula (5) can be written as in (11) and the whole process can be repeated treating each $z_{i}$ as the original $x$ in formula (11). This process terminates in finitely many steps and eventually reduces the consideration of all the elements $x$ to those of the form $a v$ where $a \in K$ and $v \in E^{0}$. This situation has been handled in case 2 above. So, this finishes the proof.

We turn to conditions characterizing faithfulness of a canonical trace now.

Theorem 3.5. Let $R$ be a positive definite $K$-algebra and $t$ a canonical, $K$-linear, $R$-valued trace on $L_{K}(E)$. Then $t$ is faithful if and only if conditions $(P)$ and $(F)$ hold where

(F) $t(v)>0$ for all vertices $v$.

Proof. If $t$ is faithful then $t$ is positive so $(\mathrm{P})$ holds. Condition (F) clearly holds as well.

Assume now $(\mathrm{P})$ and $(\mathrm{F})$. By Theorem 3.4, the trace $t$ is positive. Since $R$ is positive definite, to show that $t$ is faithful it is sufficient to show that $t\left(x x^{*}\right)=0$ implies that $x=0$ for any $x \in L_{K}(E)$ by Lemma 2.1 . The proof follows the stages of the proof of Theorem 3.4, so the labels of the formulas and notation refer to those used in the proof of Theorem 3.4. Writing $x$ as $\sum_{i=1}^{n} x_{i}$ as in formula (1), we have that $t\left(x x^{*}\right)=\sum_{i=1}^{n} t\left(x_{i} x_{i}^{*}\right)=0$. Since $t\left(x_{i} x_{i}^{*}\right) \geq 0$ because $t$ is positive, we have that $t\left(x_{i} x_{i}^{*}\right)=0$ for every $i=1, \ldots, n$ by the assumption that $R$ is positive definite. Thus, it is sufficient to prove that each $x_{i}$ is zero. So, it is sufficient to consider $x$ which has the form as in formula (21). For such $x, t\left(x x^{*}\right)=t\left(y y^{*}\right)$ where $y$ is as in (3). Since $x=p y q^{*}$, to show that $x$ is zero, it is sufficient to show that $y$ is zero. So, it is sufficient to consider $x$ to be as in (3).

For an element $x=\sum_{j=1}^{l} a_{j} r_{j} r_{j}^{*}$ with $\mathbf{s}\left(r_{j}\right)=v$ for all $j=1, \ldots, l$ as in (3)), consider again cases 1, 2, and 3 as in the proof of Theorem 3.4. In case 1, write $x$ as in (44). The terms $x_{i}, i=1, \ldots, m$ are such that $0=t\left(x x^{*}\right)=\sum_{i=1}^{m} t\left(x_{i} x_{i}^{*}\right)$. Since $R$ is positive definite and $t\left(x_{i} x_{i}^{*}\right) \geq 0$, we have that $t\left(x_{i} x_{i}^{*}\right)=0$ for all $i$. Thus, to show that $x=0$ it is sufficient to 
show that $x_{i}=0$ for every $i$. If $z_{i}, i=1, \ldots, m$, are as in (5), then $x_{i}=e_{i} z_{i} e_{i}^{*}$. Thus, to show that $x_{i}=0$ for all $i$, it is sufficient to show that $z_{i}=0$ for all $i$.

In case $2, x=a_{1} v$ where $a_{1} \in K$ and $v \in E^{0}$. If $a_{1} \neq 0$ then $0=t\left(a_{1} a_{1}^{*} v\right)=a_{1} a_{1}^{*} t(v)$ implies that $t(v)=0$ which contradicts $(\mathrm{F})$. Thus $a_{1}=0$ and so $x=a_{1} v=0$.

In case 3 , write $x$ as

$$
x=\sum_{i=1}^{m} \sum_{k=1}^{m_{i}} a_{i k} e_{i} r_{i k} r_{i k}^{*} e_{i}^{*}+a_{l} v
$$

where $e_{i}, i=1, \ldots, m$ is the list of all edges that are the first in paths $r_{j}, j=1, \ldots, l-1$ without repetition and $a_{l} \neq 0$ (otherwise $x$ falls under case 1 ). Also as in the proof of Theorem 3.4, let

$$
y=\sum_{i=1}^{m} y_{i} \quad \text { for } \quad y_{i}=\sum_{k=1}^{m_{i}} a_{i k} e_{i} r_{i k} r_{i k}^{*} e_{i}^{*}+a_{l} e_{i} e_{i}^{*}
$$

and note that $0=t\left(x x^{*}\right) \geq t\left(y y^{*}\right)=\sum_{i=1}^{m} t\left(y_{i} y_{i}^{*}\right) \geq 0$ by part (2) of Lemma 3.3 and so $\sum_{i=1}^{m} t\left(y_{i} y_{i}^{*}\right)=0$. Since $R$ is positive definite and $t\left(y_{i} y_{i}^{*}\right) \geq 0$, we have that $t\left(y_{i} y_{i}^{*}\right)=0$ for all $i=1, \ldots, m$. By part (1) of Lemma 3.3, the elements

$$
z_{i}=\sum_{k=1}^{m_{i}} a_{i k} r_{i k} r_{i k}^{*}+a_{l} \mathbf{r}\left(e_{i}\right)
$$

are such that $0=t\left(y_{i} y_{i}^{*}\right)=t\left(z_{i} z_{i}^{*}\right)$. We claim that showing $z_{i}=0$ for all $i=1, \ldots, m$ is sufficient to show that $x=0$. Indeed, if $z_{i}=0$, then $y_{i}=e_{i} z_{i} e_{i}^{*}=0$ as well and so $\sum_{k=1}^{m_{i}} a_{i k} e_{i} r_{i k} r_{i k}^{*} e_{i}^{*}=-a_{l} e_{i} e_{i}^{*}$. Thus,

$$
x=a_{l} v-\sum_{i=1}^{m} a_{l} e_{i} e_{i}^{*}=a_{l}\left(v-\sum_{i=1}^{m} e_{i} e_{i}^{*}\right) \Rightarrow x x^{*}=a_{l} a_{l}^{*}\left(v-\sum_{i=1}^{m} e_{i} e_{i}^{*}\right) .
$$

Since $t\left(x x^{*}\right)=0$, we have that $a_{l} a_{l}^{*} t\left(v-\sum_{i=1}^{m} e_{i} e_{i}^{*}\right)=0$. This implies $t\left(v-\sum_{i=1}^{m} e_{i} e_{i}^{*}\right)=0$ because $a_{l} \neq 0$. Note that $v$ is not a sink since $m>0$. If $\left\{e_{1}, \ldots, e_{m}\right\}=\mathbf{s}^{-1}(v)$ then $v$ is regular, $v=\sum_{i=1}^{m} e_{i} e_{i}^{*}$ by (CK2) and so $x=0$. If $\left\{e_{1}, \ldots, e_{m}\right\} \subsetneq \mathbf{s}^{-1}(v)$, there is an edge $f$ different from $e_{1}, \ldots, e_{m}$ with $v=\mathbf{s}(f)$. The element $v-f f^{*}-\sum_{i=1}^{m} e_{i} e_{i}^{*}$ is a projection (selfadjoint idempotent) and so it is positive. Since $t$ is positive, $v \geq f f^{*}+\sum_{i=1}^{m} e_{i} e_{i}^{*}$ implies that $t(v) \geq t\left(f f^{*}\right)+\sum_{i=1}^{m} t\left(e e^{*}\right)$. Since $(\mathrm{F})$ holds, $t\left(f f^{*}\right)=t(\mathbf{r}(f))>0$ and so

$$
t(v) \geq t\left(f f^{*}\right)+\sum_{i=1}^{m} t\left(e_{i} e_{i}^{*}\right)>\sum_{i=1}^{m} t\left(e_{i} e_{i}^{*}\right) .
$$

This contradicts $0=t\left(v-\sum_{i=1}^{m} e_{i} e_{i}^{*}\right)=t(v)-\sum_{i=1}^{m} t\left(e_{i} e_{i}^{*}\right)$.

Thus, both case 1 and case 3 reduce to the consideration of elements $z_{i}$ as in (5). The expression in (5) can be written as an expression in (11) again and the whole process can be repeated. In every step the lengths of the paths in formula (5) are shorter than the lengths of the corresponding paths in (3). Thus the process terminates in finitely many steps and eventually reduces to the consideration of the elements of the form $a v$ where $a \in K$ and $v \in E^{0}$, handled in case 2 . Thus, we have that $t\left(x x^{*}\right)=0$ implies that $x=0$ for any $x$.

Note that under assumptions of Theorem 3.5, $K$ is positive definite by Proposition 2.2 and then so is $L_{K}(E)$ by [5, Proposition 2.4].

Theorems 3.4 and 3.5 imply the following result. 
Theorem 3.6. Let $R$ be an involutive $K$-algebra. The correspondence $\tau$ from Proposition 2.7 is such that it induces a bijective correspondence between

(1) positive, $R$-valued graph traces on $E$ and

(2) positive, canonical, $K$-linear, $R$-valued traces on $L_{K}(E)$.

If $R$ is positive definite, the correspondence $\tau$ is such that it induces a bijective correspondence between

(1) faithful, $R$-valued graph traces on $E$ and

(2) faithful, canonical, $K$-linear, $R$-valued traces on $L_{K}(E)$.

Proof. Let us recall that $t_{\delta}$ denotes the unique extension of an $R$-valued graph trace $\delta$ on $E$ to a canonical, $K$-linear, $R$-valued trace on $L_{K}(E)$ in the proof of Proposition 2.7. Theorem 3.4 implies that if such graph trace $\delta$ is positive, then $t_{\delta}$ is positive as well. Conversely, if $t$ is a positive, canonical, $K$-linear, $R$-valued trace, then condition $(\mathrm{P})$ holds. Since condition $(\mathrm{P})$ implies $(\mathrm{P})_{\delta}$ for the restriction of $t$ to vertices, the claim follows.

Similarly, if $R$ is positive definite and a graph trace $\delta$ is faithful, then $t_{\delta}$ is faithful as well by Theorem 3.5. The converse clearly follows since condition $(\mathrm{F})$ implies $(\mathrm{F})_{\delta}$ for the restriction of $t$ to vertices.

The assumption that $R$ is positive definite is necessary in Theorem 3.5 and the second part of Theorem [3.6. [12, Example 34] can be used to demonstrate this. In this example, the Leavitt path algebra of the graph with two vertices $v$ and $w$ and one edge $e$ from $v$ to $w$ is considered over the field of complex numbers with the identity involution. Mapping both vertices to 1 defines a faithful, $\mathbb{C}$-valued graph trace $\delta$ on $E$. Since $\delta$ is positive, it extends to a positive, canonical, $\mathbb{C}$-linear, $\mathbb{C}$-valued trace $t$ by Theorem 3.6 and $t$ is such that conditions $(\mathrm{P})$ and $(\mathrm{F})$ are fulfilled. However, $t$ is not faithful since $v-w=(v+i w)(v+i w)^{*} \geq 0$, $t(v-w)=1-1=0$ and $v-w \neq 0$.

Theorem 3.6 has the following corollary.

Corollary 3.7. The following conditions are equivalent for any positive definite field $K$.

(1) There is a faithful, canonical, $K$-linear, $K$-valued trace on $L_{K}(E)$.

(2) There is a faithful, canonical, $K$-valued trace on $L_{K}(E)$.

(3) There is a faithful, $K$-linear, $K$-valued trace on $L_{K}(E)$.

(4) There is a faithful, $K$-valued trace on $L_{K}(E)$.

(5) There is a faithful, $K$-valued graph trace on $E$.

Proof. The implications $(1) \Rightarrow(2) \Rightarrow(4)$ and $(1) \Rightarrow(3) \Rightarrow(4)$ are tautologies. Condition (4) implies (5) since the restriction of a trace as in (4) to $E^{0}$ is a graph trace as in (5). Finally, (5) implies (1) since every graph trace as in (5) extends to a trace as in (1) by Theorem 3.6 .

If any of the equivalent conditions (1)-(5) hold for a positive definite field $K$, we say that $L_{K}(E)$ admits a faithful trace.

We conclude this section with another corollary of Theorem 3.6. Namely, in [14, Proposition 3.9], it has been shown that there is a bijective correspondence between faithful, $\mathbb{C}$-valued graph traces on a countable, row-finite graph $E$ and faithful, semifinite, lower semicontinuous, gauge invariant, $\mathbb{C}$-valued traces on $C^{*}(E)$. In [14], a trace $t$ on a $C^{*}$-algebra $A$ is defined as an additive map on the positive cone $A^{+}$of $A$ taking values in $[0, \infty]$ such that $t(a x)=a t(x)$ for a nonnegative real number $a$ and $x \in A^{+}$and $t\left(x x^{*}\right)=t\left(x^{*} x\right)$ for all $x \in A$. 
To avoid confusion with our definition of a trace, we shall refer to such map as a $C^{*}$-trace. Clearly every positive, $\mathbb{C}$-linear trace on $A$ is a $C^{*}$-trace.

Recall that every element $x=a+i b$ of a $C^{*}$-algebra $A$ where $a$ and $b$ are the real and imaginary parts of $x$ (see [11, page 105]), can be written as a $\mathbb{C}$-linear combination $x=\left(a^{+}-a^{-}\right)+i\left(b^{+}-b^{-}\right)$where $a^{+}, a^{-}, b^{+}, b^{-}$are positive elements such that $a^{+} a^{-}=$ $a^{-} a^{+}=b^{+} b^{-}=b^{-} b^{+}=0$ and this representation is unique ([11, Corollary 4.2.4]). Thus, every $C^{*}$-trace defined on the positive cone of $A$ can be extended to $A$ by letting $t(x)=$ $t\left(a^{+}\right)-t\left(a^{-}\right)+i\left(t\left(b^{+}\right)-t\left(b^{-}\right)\right)$. It is straightforward to check that this extension is $\mathbb{C}$-linear and positive. Without any danger of confusion, we shall refer to this extension of a $C^{*}$-trace as a $C^{*}$-trace as well.

A $C^{*}$-trace $t$ on a $C^{*}$-algebra $A$ is defined to be faithful if $t\left(x x^{*}\right)=0$ implies that $x=0$. This condition is equivalent to the one we use to define a faithful additive map by Lemma 2.1 because the complex-conjugate involution is positive definite and every $C^{*}$-algebra is proper. A $C^{*}$-trace $t$ on $A$ is semifinite if the set of elements of $A^{+}$with finite trace is norm dense in $A^{+}$. A $C^{*}$-trace $t$ on $A$ is lower semicontinuous if $t\left(\lim _{n \rightarrow \infty} a_{n}\right) \leq \liminf _{n \rightarrow \infty} t\left(a_{n}\right)$ for all norm convergent sequences $a_{n}$ in $A^{+}$.

If $\left\{S_{e}, p_{v} \mid e \in E^{1}, v \in E^{0}\right\}$ is a Cuntz-Krieger $E$-family for a graph $C^{*}$-algebra $C^{*}(E)$ (see [7], [16] or [14 for example), the gauge action $\lambda$ on the unit sphere $S^{1}$ is given by $\lambda_{z}\left(S_{p} S_{q}^{*}\right)=z^{|p|-|q|} S_{p} S_{q}^{*}$ for $z \in S^{1}$ and paths $p$ and $q$. A $C^{*}$-trace $t$ on $C^{*}(E)$ is gauge invariant if $t\left(\lambda_{z} S_{p} S_{q}^{*}\right)=t\left(S_{p} S_{q}^{*}\right)$ for every complex number $z$ of unit length. Since such $t$ is $\mathbb{C}$-linear, this condition is equivalent to

$$
t\left(S_{p} S_{q}^{*}\right)=z^{|p|-|q|} t\left(S_{p} S_{q}^{*}\right) \text { for all complex numbers } z \text { of unit length }
$$

and all paths $p$ and $q$. Thus, if a $C^{*}$-trace is gauge invariant in the sense of Definition 2.5, then it is gauge invariant in this sense. Our next result, Corollary 3.8, shows that the converse holds for faithful, semifinite, lower semicontinuous $C^{*}$-traces on $C^{*}(E)$ if $E$ is countable. Corollary 3.8 also shows that it is not necessary to assume that $E$ is row-finite in [14, Proposition 3.9].

Corollary 3.8. Let $E$ be a countable graph and consider $\mathbb{C}$ with the complex-conjugate involution. The following sets are in bijective correspondences.

(1) The set of faithful, $\mathbb{C}$-valued graph traces on $E$,

$(2)$ the set of faithful, gauge invariant, $\mathbb{C}$-linear, $\mathbb{C}$-valued traces on $L_{\mathbb{C}}(E)$, and

(3) the set of faithful, semifinite, lower semicontinuous, gauge invariant $C^{*}$-traces on $C^{*}(E)$.

A faithful, semifinite, lower semicontinuous $C^{*}$-trace on $C^{*}(E)$ satisfies (GI) if and only if it is gauge invariant (in the sense of Definition 2.5).

Proof. Since the complex-conjugate involution is positive definite, the sets (1) and (2) are in a bijective correspondence by Theorem 3.6.

In [14], $E$ is assumed to be countable and row-finite. By [14, Lemma 3.2], every semifinite $C^{*}$-trace on $C^{*}(E)$ is such that the trace of an element of $L_{\mathbb{C}}(E)$ is finite but the assumption that $E$ is row-finite is not used it the proof. Thus, every $C^{*}$-trace as in $(3)$ restricts to a graph trace as in (1) by the proof of [14, Lemma 3.2].

Thus, it remains to show that every trace as in (2) extends to a trace as in (3). The proof of [14, Proposition 3.9] shows this claim for $E$ countable and row-finite. The assumption that $E$ is row-finite is used only when invoking the Gauge Invariant Uniqueness Theorem for row-finite graphs from [8]. This theorem has been shown for countable graphs in [7, 
Theorem 2.1] so we need to require just that $E$ is countable. The proof of [14, Proposition 3.9 ] shows that $t$ satisfies condition (GI). However, since $t$ is canonical on $L_{\mathbb{C}}(E)$ and $\mathbb{C}$ has characteristic zero, $t$ is gauge invariant by Proposition 2.4 .

To prove the last sentence of this corollary, it is sufficient to prove that a faithful, semifinite, lower semicontinuous, $C^{*}$-trace on $C^{*}(E)$ which satisfies $(\mathrm{GI})$ is canonical. If $t$ is such a trace, the restriction of $t$ on the vertices is a faithful graph trace $\delta$. The extension of $\delta$ to $L_{\mathbb{C}}(E)$ is a canonical trace whose extension to $C^{*}(E)$ is $t$. Thus, $t$ is canonical.

\section{Cohn-Leavitt algebras and directly finite LeavitT path algebras}

In this section, we characterize directly finite Leavitt path algebras as exactly those Leavitt path algebras $L_{K}(E)$ for which $E$ is a no-exit graph (Theorem 4.12). The proof of this characterization involves consideration of Cohn-Leavitt algebras, the algebraic counterparts of relative graph $C^{*}$-algebras, for which we also formulate all our previous results. Lastly, we compare the classes of locally noetherian, directly finite and those Leavitt path algebras which admit a faithful trace.

Recall that a unital ring is directly (or Dedekind) finite if $x y=1$ implies that $y x=1$ for all $x$ and $y$. The involutive version of this definition is the following: a ring is finite if $x x^{*}=1$ implies $x^{*} x=1$ for all $x$. This terminology comes from operator theory and should not be confused with rings having finite cardinality. In the rest of the paper, when we refer to a $*$-ring or a $*$-algebra being finite, we assume the finiteness in this sense.

We adapt finiteness and direct finiteness to non-unital rings with local units. Recall that a ring $R$ has local units if for every finite set $x_{1}, \ldots, x_{n} \in R$ there is an idempotent $u$ such that $x_{i} u=u x_{i}=x_{i}$ for all $i=1, \ldots, n$.

Definition 4.1. A ring with local units $R$ is said to be directly finite if for every $x, y \in R$ and an idempotent element $u \in R$ such that $x u=u x=x$ and $y u=u y=y$, we have that

$$
x y=u \text { implies } y x=u \text {. }
$$

A *-ring with local units $R$ is said to be finite if for every $x \in R$ and an idempotent $u \in R$ such that $x u=u x=x$, we have that

$$
x x^{*}=u \text { implies } x^{*} x=u .
$$

Condition $x x^{*}=u$ implies that $u$ is a projection (selfadjoint idempotent) since $u^{*}=\left(x x^{*}\right)^{*}=$ $x x^{*}=u$. Thus, $x^{*} u=u x^{*}=x^{*}$ as well.

If $R$ is a unital, directly finite ring, then it is directly finite in the locally-unital sense as well. Indeed, assuming that $x y=u$ for an idempotent element $u$ with $x u=u x=x$ and $y u=u y=y$ we have that $(x+1-u)(y+1-u)=x y+1-u=1$. This implies that $1=(y+1-u)(x+1-u)=y x+1-u$ and from this it follows that $y x=u$. Similarly, if $R$ is a unital, finite $*$-ring, then it is finite in the locally-unital sense as well.

The fact that the existence of a faithful trace on a (unital) von Neumann algebra implies its finiteness is well known and widely used. The arguments proving this fact easily generalize to any unital $*$-ring with a faithful trace. We note this fact for locally unital rings. In fact, as the next proposition shows, a more general claim holds: any ring with local units and a trace which is injective on idempotents is directly finite.

Proposition 4.2. If $R$ is a ring with local units and there is a trace on $R$ which is injective on idempotent elements, then $R$ is directly finite. 
If $R$ is a *-ring with local units and there is a trace on $R$ which is injective on projections, then $R$ is finite. In particular, a*-ring with local units and a faithful trace is finite.

Proof. Let $R$ be a ring with local units, $t$ a trace on $R$ which is injective on idempotents, and let $x, y$ be in $R$ such that $x u=u x=x$ and $y u=u y=y$ for some idempotent $u$. If $x y=u$, then $u-y x$ is an idempotent since $(u-y x)(u-y x)=u-y x-y x+y x y x=$ $u-y x-y x+y u x=u-y x$. Then $t(u-y x)=t(x y-y x)=0$ which implies that $u-y x=0$ since $t$ is injective on idempotents. Thus $y x=u$.

The second sentence is proven analogously and the third is a consequence of the second.

Note that $L_{K}(E)$ is a ring with local units. Indeed for any $x_{i}$ in $L_{K}(E), i=1, \ldots, n$ which can be represented using paths $p_{i j}, q_{i j}$ and $a_{i j} \in K, j=1, \ldots, n_{i}$ as $x_{i}=\sum_{j=1}^{n_{i}} a_{i j} p_{i j} q_{i j}^{*}$, we have that the sum $u$ of all vertices that are sources of all paths $p_{i j}$ and $q_{i j}$ for all $i=1, \ldots, n$ and $j=1, \ldots, n_{i}$ is an idempotent with $x_{i} u=x_{i}=u x_{i}$.

The direct finiteness of a Leavitt path algebra forces the underlying graph to be no-exit. This has been shown to hold in [6, Proposition 3.1] for Leavitt path algebras of finite graphs. The proof of part (6) of [12, Proposition 29] shows this claim for any Leavitt path algebra but since [12, Proposition 29 (6)] is worded in a different set up, we list the proof below.

Proposition 4.3. If $L_{K}(E)$ is (directly) finite, then $E$ is no-exit.

Proof. Since direct finiteness implies finiteness, it is sufficient to show the claim assuming that $L_{K}(E)$ is finite. In this case, assume that $E$ has a cycle $p$ with an exit $e$. We also may assume that $\mathbf{s}(p)=\mathbf{r}(p)=\mathbf{s}(e)$, and we denote this vertex by $v$. Let $w=\mathbf{r}(e)$, $x=p+\left(1-\delta_{v, w}\right) w$ and $u=v+\left(1-\delta_{v, w}\right) w$. Then we have that $x u=u x=x$ and that $x^{*} x=p^{*} p+\left(1-\delta_{v, w}\right) w=v+\left(1-\delta_{v, w}\right) w=u$. By finiteness, we then have that $v+\left(1-\delta_{v, w}\right) w=u=x x^{*}=p p^{*}+\left(1-\delta_{v, w}\right) w$. Hence, $v=p p^{*}$. But then $0=e^{*} p p^{*}=e^{*} v=e^{*}$ which is a contradiction. Thus, $p$ cannot have an exit.

Our goal is to prove that the converse of Proposition 4.3 holds. This has been proven for Leavitt path algebras of finite graphs in [6, Theorem 3.3]. Thus, if we can "localize" our main claim, i.e. reduce the consideration of the general case to a Leavitt path algebra of a finite subgraph and then use [6, Theorem 3.3], then we would achieve our goal. In particular, assuming that a graph $E$ is no-exit and considering $x, y \in L_{K}(E)$ such that $x y=u$ for some local unit $u$, we aim to consider a finite subgraph $F$ generated by the vertices and edges of just those paths that appear in representations of $x, y$ and $u$. The problem is that the subgraph $F$ defined in this way may not be complete in the sense of [2, Definition 9.7] and so $L_{K}(F)$ may not be a subalgebra of $L_{K}(E)$. However, we show that this impediment can be avoided by considering Cohn-Leavitt algebras of [3]. Namely, we can consider appropriate finite subgraph $F$ such that the Cohn-Leavitt algebra of $F$ is a subalgebra of $L_{K}(E)$ and we can adapt [6, Theorem 3.3] to Cohn-Leavitt algebras of finite graphs. This approach requires us to recall the definition of Cohn-Leavitt algebras and demonstrate some preliminaries.

Cohn-Leavitt algebras are obtained by requiring the (CK2) axiom to hold just for a portion of regular vertices, not necessarily all of them. More precisely, if $S$ is a subset of regular vertices, the Cohn-Leavitt algebra $C L_{K}(E, S)$ of $E$ and $S$ over $K$ is a free $K$-algebra generated by the sets $E^{0} \cup E^{1} \cup\left\{e^{*} \mid e \in E^{1}\right\}$ with relations (V), (E1), (E2), (CK1) and

(SCK2) $v=\sum_{e \in \mathbf{s}^{-1}(v)} e e^{*}$, for every vertex $v \in S$.

For the rest of the paper, $R(E)$ denotes the set of the regular vertices of $E$ and $S$ a subset of $R(E)$. If $S$ is empty, the Cohn-Leavitt algebra $C L_{K}(E, S)$ is a Cohn path algebra and we 
write $C L_{K}(E, \emptyset)$ as $C_{K}(E)$. If $S$ is equal to $R(E)$ then $C L_{K}(E, S)$ is a Leavitt path algebra and we write $C L_{K}(E, R(E))$ as $L_{K}(E)$.

The $C^{*}$-analog of Cohn-Leavitt algebras preceded the consideration of Cohn-Leavitt algebras. In [13], Muhly and Tomforde introduced the relative graph $C^{*}$-algebra $C^{*}(E, S)$ of a graph $E$ and $S \subseteq R(E)$ as the $C^{*}$-algebra generated by a universal Cuntz-Krieger $(E, S)$ family, obtained by replacing the (CK2) axiom of a Cuntz-Krieger $E$-family by the (SCK2) axiom ([13, Definition 3.5]). In [3], Cohn-Leavitt algebras are introduced for a more general class of graphs, called separated graphs, than those considered in this paper. The graphs considered in this paper correspond to those from [3] with trivial separation.

If $E$ is a countable graph, [13, Theorem 3.7] shows that the relative graph $C^{*}$-algebra $C^{*}(E, S)$ is canonically isomorphic to the graph $C^{*}$-algebra $C^{*}\left(E_{S}\right)$ of a suitable graph $E_{S}$. In the paragraph preceding Lemma 4.8, we review this construction and adapt it to show that $C L_{K}(E, S)$ is isomorphic to $L_{K}\left(E_{S}\right)$ for any graph $E$. Thus, the class of Cohn-Leavitt algebras is not larger than the class of Leavitt path algebras as it first may seem. Still, considering Cohn-Leavitt algebras is an elegant way to unite considerations of both Cohn path and Leavitt path algebras. Because of this, we also formulate the results of previous sections in terms of Cohn-Leavitt algebras. As a consequence, each results is readily applicable to a Cohn path or any other Cohn-Leavitt algebra without referring to the construction of the graph $E_{S}$ or the isomorphism $C L_{K}(E, S) \cong L_{K}\left(E_{S}\right)$.

Using relations $(\mathrm{V}),(\mathrm{E} 1),(\mathrm{E} 2)$ and $(\mathrm{CK} 1)$, every nonzero element of $C L_{K}(E, S)$ can be represented as a finite $K$-linear combination of elements of the form $p q^{*}$ where $p$ and $q$ are paths. Thus, the involution $*$ from $K$ extends to an involution of $C L_{K}(E, S)$ by $\left(\sum_{i=1}^{n} a_{i} p_{i} q_{i}^{*}\right)^{*}=\sum_{i=1}^{n} a_{i}^{*} q_{i} p_{i}^{*}$ for paths $p_{i}$ and $q_{i}$ and $a_{i} \in K, i=1, \ldots, n$, making $C L_{K}(E, S)$ an involutive $K$-algebra.

We adapt Theorems 3.4 and 3.5 to Cohn-Leavitt algebras now.

Proposition 4.4. Let $R$ be an involutive $K$-algebra and $t$ a canonical, $K$-linear, $R$-valued trace on $C L_{K}(E, S)$. The trace $t$ is positive if and only if

(P) $\quad t\left(v-\sum_{e \in I} \mathbf{r}(e)\right) \geq 0$ for all vertices $v$ and finite subsets $I$ of $\mathbf{s}^{-1}(v)$.

If $R$ is positive definite, then $t$ is faithful if and only if $(P),(F)$ and $(S F)$ hold.

(F) $t(v)>0$ for all vertices $v$.

$(\mathrm{SF}) t\left(v-\sum_{e \in \mathbf{s}^{-1}(v)} \mathbf{r}(e)\right)>0$ for all regular vertices $v$ not in $S$.

Proof. Note that axiom (CK2) was not used in the proofs of Theorems 3.4 and any of its preliminary results. Thus, the proof of Theorem 3.4 directly transfers to the proof of the first part of the claim.

The proof of Theorem 3.5 also directly carries over to the proof of the second part of the claim except for the following step of the proof which requires the use of $(\mathrm{SF})$ : assuming that $t$ is such that $(\mathrm{P}),(\mathrm{F})$, and $(\mathrm{SF})$ hold,

$$
t\left(v-\sum_{e \in I} e e^{*}\right)=0 \text { implies } v=\sum_{e \in I} e e^{*}
$$

for any vertex $v$ and any finite set $I \subseteq \mathbf{s}^{-1}(v)$. To prove this step, note first that the claim trivially holds if $v$ is a sink or $I$ is empty since the assumption $t(v)=0$ is false by $(\mathrm{F})$. Thus we can assume that $v$ is not a sink and $I$ is nonempty. In this case, assume that $t\left(v-\sum_{e \in I} e e^{*}\right)=0$. Condition (SF) implies that $v \notin R(E) \backslash S$ or $I \subsetneq \mathbf{s}^{-1}(v)$. With these restrictions, we either have $I=\mathbf{s}^{-1}(v)$ and $v \in S$ or $I \subsetneq \mathbf{s}^{-1}(v)$. If $v \in S$ and $I=\mathbf{s}^{-1}(v)$, 
$v=\sum_{e \in I} e e^{*}$ by (SCK2). If $I \subsetneq \mathbf{s}^{-1}(v)$, there is an edge $f \in \mathbf{s}^{-1}(v) \backslash I$. The element $v-f f^{*}-\sum_{e \in I} e e^{*}$ is selfadjoint and is easily seen to be idempotent using just (V), (E1), (E2) and (CK1). So, it is positive. Thus, we have that

$$
t(v) \geq t\left(f f^{*}\right)+\sum_{e \in I} t\left(e e^{*}\right)>\sum_{e \in I} t\left(e e^{*}\right)
$$

by positivity of $t$ and condition $(\mathrm{F})$. This contradicts $0=t\left(v-\sum_{e \in I} e e^{*}\right)=t(v)-\sum_{e \in I} t\left(e e^{*}\right)$ so the case $I \subsetneq \mathbf{s}^{-1}(v)$ cannot happen. Thus $v \in S$ and $I=\mathbf{s}^{-1}(v)$ in which case $v=$ $\sum_{e \in I} e e^{*}$. The rest of the proof of Theorem 3.5 directly transfers to the proof of the present claim.

The proofs of Proposition 2.7 and Theorem 3.6 can also be transfered directly to CohnLeavitt setting after adjusting the definition of a graph trace as follows.

Definition 4.5. If $R$ is a ring, an $R$-valued graph trace on $E$ relative to $S$ is a map $\delta: E^{0} \rightarrow R$ such that

$(\mathrm{SCK} 2)_{\delta} \delta(v)=\sum_{e \in \mathbf{s}^{-1}(v)} \delta(\mathbf{r}(e))$ for all vertices $v$ in $S$.

A graph trace on $E$ relative to $R(E)$ is simply called a graph trace on $E$.

If $R$ is an involutive $K$-algebra and $\delta$ a graph trace on $E$ relative to $S$, then $\delta$ is positive if condition $(\mathrm{P})_{\delta}$ holds. If $\delta$ is positive, then $\delta$ is faithful if $(\mathrm{F})_{\delta}$ and $(\mathrm{SF})_{\delta}$ hold for

$(\mathrm{SF})_{\delta} \delta(v)>\sum_{e \in \mathbf{s}^{-1}(v)} \delta(\mathbf{r}(e))$ for all regular vertices $v$ not in $S$.

Proposition 4.6. Proposition [2.7 and Theorem [3.6 hold for $C L_{K}(E, S)$ after every appearance of "graph trace" is replaced by "graph trace relative to $S$ ".

Proof. By considering graph traces relative to $S$ instead of graph traces, using (SCK2) instead of $(\mathrm{CK} 2)$ and $(\mathrm{SCK} 2)_{\delta}$ instead of $(\mathrm{CK} 2)_{\delta}$, we obtain the proofs of [12, Theorem 28] and Proposition 2.7 adjusted to Cohn-Leavitt algebras. As a consequence of this and Proposition 4.4, Theorem 3.6, adjusted appropriately, holds for $C L_{K}(E, S)$.

Our next goal is to adapt the construction from [13, Theorem 3.7] to show that any CohnLeavitt algebra $C L_{K}(E, S)$ is $*$-isomorphic to the Leavitt path algebra $L_{K}\left(E_{S}\right)$ of a suitable graph $E_{S}$ defined via $E$ and $S$. Recall that a homomorphism $f$ of $*$-rings is said to be a *-homomorphism if $f\left(x^{*}\right)=f(x)^{*}$ for every $x$ in the domain and that a $*$-isomorphism is an isomorphism which is also a $*$-homomorphism. Also recall that the universal property of Leavitt path algebras states that if $R$ is a $K$-algebra which contains a set $\left\{a_{v}, b_{e}, c_{e^{*}} \mid v \in\right.$ $\left.E^{0}, e \in E^{1}\right\}$ such that $a_{v}, b_{e}, c_{e^{*}}$ satisfy axioms (V), (E1), (E2), (CK1), and (CK2) (such set is called a Leavitt E-family) then there is a unique $K$-algebra homomorphism $f: L_{K}(E) \rightarrow R$ such that $f(v)=a_{v}, f(e)=b_{e}$, and $f\left(e^{*}\right)=c_{e^{*}}$ for all $v \in E^{0}$ and $e \in E^{1}$ (see [2, Remark 2.11] for example). The next lemma explores the requirements for such homomorphism $f$ to be a $*$-homomorphism.

Lemma 4.7. For every involutive $K$-algebra $R$ with a Leavitt $E$-family $\left\{a_{v}, b_{e}, c_{e^{*}} \mid v \in E^{0}, e \in\right.$ $\left.E^{1}\right\}$ such that $a_{v}^{*}=a_{v^{*}}$ and $b_{e}^{*}=c_{e^{*}}$, there is a unique K-algebra *-homomorphism $f$ : $L_{K}(E) \rightarrow R$ such that $f(v)=a_{v}, f(e)=b_{e}$, (thus $f\left(e^{*}\right)=c_{e^{*}}$ ) for all $v \in E^{0}$ and $e \in E^{1}$.

Proof. Since $\left\{a_{v}, b_{e}, c_{e^{*}} \mid v \in E^{0}, e \in E^{1}\right\}$ is a Leavitt $E$-family, there is a unique $K$-algebra homomorphism $f: L_{K}(E) \rightarrow R$ such that $f(v)=a_{v}, f(e)=b_{e}$, and $f\left(e^{*}\right)=c_{e^{*}}$. We claim that under assumption that $a_{v}^{*}=a_{v}$ and $b_{e}^{*}=c_{e^{*}}$, the map $f$ is a $*$-homomorphism. 
Since $f$ is additive and $K$-linear, it is sufficient to prove that $f\left(x^{*}\right)=f(x)^{*}$ if $x=p q^{*}$ where $p$ and $q$ are paths with $\mathbf{r}(p)=\mathbf{r}(q)$. The condition $a_{v}^{*}=a_{v}$ proves this statement for $|p|=|q|=0$ and the condition $b_{e}^{*}=c_{e^{*}}$ implies that $f(e)^{*}=f\left(e^{*}\right)$ for every edge $e$. Assuming that the statement holds for any path $p$ with $|p|<n$ and $q$ with $|q|=0$, let us prove it if $p$ has length $n$ and $q$ length 0 . In this case $p=e r$ for some edge $e$ and path $r$ with $\mathbf{r}(e)=\mathbf{s}(r)$ and $|r|<n$ so that $f(r)^{*}=f\left(r^{*}\right)$ by the induction hypothesis and $p q^{*}=e r$. Thus $f\left(p q^{*}\right)^{*}=$ $f(e r)^{*}=(f(e) f(r))^{*}=f(r)^{*} f(e)^{*}=f\left(r^{*}\right) f\left(e^{*}\right)=f\left(r^{*} e^{*}\right)=f\left((e r)^{*}\right)=f\left(\left(p q^{*}\right)^{*}\right)$.

Now, assuming the statement for $p q^{*}$ with $|q|<m$, let us prove it for $p q^{*}$ with $|q|=m$. In this case $q=e r$ for some edge $e$ and path $r$ with $\mathbf{r}(e)=\mathbf{s}(r)$ and $|r|<m$ and so $f\left(p q^{*}\right)^{*}=f\left(p r^{*} e^{*}\right)^{*}=\left(f\left(p r^{*}\right) f\left(e^{*}\right)\right)^{*}=f\left(e^{*}\right)^{*} f\left(p r^{*}\right)^{*}=f(e) f\left(r p^{*}\right)=f\left(e r p^{*}\right)=$ $f\left(\left(p r^{*} e^{*}\right)^{*}\right)=f\left(\left(p q^{*}\right)^{*}\right)$.

We shall use Lemma 4.7 to show that a Cohn-Leavitt algebra $C L_{K}(E, S)$ is $*$-isomorphic to the Leavitt path algebra $L_{K}\left(E_{S}\right)$ where $E_{S}$ is the graph obtained from $E$ and $S$ as in [13, Theorem 3.7]. First, we recall the construction of $E_{S}$ from [13, Definition 3.6] and the map $\phi$ defined on the vertices, edges and ghost edges of $E_{S}$ with values in $C L_{K}(E, S)$ which creates a Leavitt $E_{S}$-family in $C L_{K}(E, S)$.

Let $E_{S}^{0}=E^{0} \cup\left\{v^{\prime} \mid v \in R(E) \backslash S\right\}$ and $E_{S}^{1}=E^{1} \cup\left\{e^{\prime} \mid e \in E^{1}\right.$ with $\left.\mathbf{r}(e) \in R(E) \backslash S\right\}$. The maps $\mathbf{s}$ and $\mathbf{r}$ in $E_{S}$ are the same as in $E$ on $E^{1}$ and such that $\mathbf{s}\left(e^{\prime}\right)=\mathbf{s}(e)$ and $\mathbf{r}\left(e^{\prime}\right)=\mathbf{r}(e)^{\prime}$ for any added edge $e^{\prime}$.

Define $\phi$ on the vertices of $E_{S}$ by $\phi(v)=v$ if $v \notin R(E) \backslash S, \phi(v)=\sum_{e \in \mathbf{s}^{-1}(v)} e e^{*}$ if $v \in R(E) \backslash S$, and $\phi\left(v^{\prime}\right)=v-\sum_{e \in \mathbf{s}^{-1}(v)} e e^{*}$ for $v \in R(E) \backslash S$. Note that this automatically gives us $\phi(w)^{*}=\phi(w)$ for every vertex $w$ of $E_{S}$. Define $\phi$ on the edges of $E_{S}$ by $\phi(e)=e \phi(\mathbf{r}(e))$ for $e \in E^{1}$ and $\phi\left(e^{\prime}\right)=e \phi\left(\mathbf{r}(e)^{\prime}\right)$ for $e \in E^{1}$ such that $\mathbf{r}(e) \in R(E) \backslash S$. Moreover, define $\phi$ on the ghost edges of $E_{S}$ by $\phi\left(f^{*}\right)=\phi(f)^{*}$ for every edge $f$ of $E_{S}$.

Lemma 4.8. The map $\phi$ extends to a *-isomorphism $\phi: L_{K}\left(E_{S}\right) \cong C L_{K}(E, S)$.

Proof. It can be directly checked that the map $\phi$ defined as above is such that the images $\phi(w), \phi(f)$, and $\phi\left(f^{*}\right)$ for $w \in E_{S}^{0}$ and $f \in E_{S}^{1}$ satisfy (V), (E1), (E2), (CK1), and (CK2). Since $\phi(w)^{*}=\phi(w)$ for $w \in E_{S}^{0}$ and $\phi\left(f^{*}\right)=\phi(f)^{*}$ for $f \in E_{S}^{1}$, the set $\left\{\phi(w), \phi(f), \phi\left(f^{*}\right) \mid w \in E_{S}^{0}, f \in E_{S}^{1}\right\}$ satisfies the assumptions of Lemma 4.7 and so $\phi$ uniquely extends to a $K$-algebra $*$-homomorphism of $L_{K}\left(E_{S}\right)$ to $C L_{K}(E, S)$.

Note that $\phi$ is onto since the vertices, edges and ghost edges of $E$ are in the image of $\phi$. Indeed, $v=\phi\left(v+v^{\prime}\right)$ for $v \in R(E) \backslash S$ and $v=\phi(v)$ for a vertex $v \notin R(E) \backslash S$. Also, if $e \in E^{1}, e=\phi\left(e+e^{\prime}\right)$ for $\mathbf{r}(e) \in R(E) \backslash S$ and $e=\phi(e)$ otherwise. From this it follows that the ghost edges of $E^{1}$ are in the image of $\phi$ as well.

Since $\phi$ preserves the grading on the vertices, edges and ghost edges, $\phi$ is a graded homomorphism by construction. Thus $\phi$ is a monomorphism by the Graded Uniqueness Theorem ([15, Theorem 4.8]). Note that $E$ is assumed to be countable in [15] but the proof of [15, Theorem 4.8] does not use this fact.

Using Lemma 4.8 and [5, Proposition 2.4], we note that the following conditions are equivalent. [5, Proposition 2.4] states that the three conditions, analogous to the three conditions below but formulated for Leavitt path algebras, are equivalent.

(1) The involution on $K$ is positive definite.

(2) The involution on $C L_{K}(E, S)$ is positive definite for every graph $E$ and $S \subseteq R(E)$.

(3) The involution on $C L_{K}(E, S)$ is positive definite for some graph $E$ and $S \subseteq R(E)$. 
Using the implication $(1) \Rightarrow(2)$, we show the Cohn-Leavitt version of Proposition 2.2.

Corollary 4.9. If $R$ is a positive definite, involutive $K$-algebra and $t: C L_{K}(E, S) \rightarrow R$ is a faithful, $K$-linear map, then $K$ and $C L_{K}(E, S)$ are positive definite.

Proof. If $\phi$ is the isomorphism from Lemma 4.8, then the composition $t \circ \phi$ satisfies the assumption of Proposition 2.2 so $K$ is positive definite by Proposition 2.2. Then $C L_{K}(E, S)$ is positive definite by Proposition 2.2 and the implication $(1) \Rightarrow(2)$ above.

Continuing on towards proving the main result of this section, we note that any CohnLeavitt algebra is a ring with local units (to see that use the same arguments as before when considering Leavitt path algebras). Thus, the definitions of directly finite and finite locally unital rings apply to Cohn-Leavitt path algebras as well. In addition to forcing the underling graph $E$ to be no-exit, the direct finiteness of a Cohn-Leavitt path algebra $C L_{K}(E, S)$ also forces the vertices of all cycles of $E$ to be in $S$. Using Lemma 4.8 and [6, Theorem 3.3], the next result shows that these conditions are also sufficient for direct finiteness if $E$ is finite.

Proposition 4.10. If $C L_{K}(E, S)$ is (directly) finite, then the following two conditions hold.

(1) E is no-exit.

(2) If a vertex is in a cycle, then it is in $S$.

If $E$ is a finite graph, conditions (1) and (2) imply that $C L_{K}(E, S)$ is directly finite.

Proof. The proof of Proposition 4.3 demonstrates part (1) since it does not use axiom (CK2).

To show part (2), assume that a vertex $v$ is in a cycle. Then $v$ is not a sink nor it is an infinite emitter since $E$ is no-exit. Thus $v$ is regular. If $v$ is not in $S$, then $v$ is in a cycle with an exit in the graph $E_{S}$ by construction of $E_{S}$. Thus, $L_{K}\left(E_{S}\right)$ is not finite by Proposition 4.3. Since $C L_{K}(E, S)$ is $*$-isomorphic to $L_{K}\left(E_{S}\right)$ by Lemma 4.8, $C L_{K}(E, S)$ is not finite as well. This contradicts the assumption so $v$ is in $S$.

Assume now that $E$ is a finite graph satisfying (1) and (2). Conditions (1) and (2) imply that the graph $E_{S}$ is no-exit by construction of $E_{S}$. Thus, $L_{K}\left(E_{S}\right)$ is directly finite by $[6$, Theorem 3.3] and so $C L_{K}(E, S) \cong L_{K}\left(E_{S}\right)$ is directly finite as well.

The last ingredient needed for our proof of Theorem 4.12 is the construction from [3, Definition 3.4, Propositions 3.5 and 3.6]. In [3], the authors consider Cohn-Leavitt algebras of separated graphs. Since we consider non-separated (i.e. trivially separated) graphs, we present [3. Definition 3.4, Propositions 3.5 and 3.6] below assuming the trivial partition $\left\{\mathbf{s}^{-1}(v)\right\}$ for every $v$. With this restriction, [3, Definition 3.4] can be stated as follows.

Let $E$ be a graph with $S \subseteq R(E)$ and $F$ a subgraph of $E$ with $T \subseteq R(F)$. We say that $(F, T)$ is a complete subobject of $(E, S)$ if $T \subseteq S$ and the following holds.

(C) If $v \in S \cap F^{0}$ with $\mathbf{s}_{E}^{-1}(v) \cap F^{1} \neq \emptyset$ then $\mathbf{s}_{F}^{-1}(v)=\mathbf{s}_{E}^{-1}(v)$ and $v \in T$.

If $T=R(F)$ and $S=R(E)$, this agrees with the definitions of a complete subgraph for row-finite graphs from [4, Section 3] and for countable graphs from [2, Definition 9.7]. Note that conditions $T \subseteq S$ and (C) imply that $T=S \cap\left\{v \in F^{0} \mid \mathbf{s}_{E}^{-1}(v) \cap F^{1} \neq \emptyset\right\}$. Indeed, every vertex $v \in T$ is necessarily in $S$ and it emits (finitely many) edges in $F$ so that $\mathbf{s}_{E}^{-1}(v) \cap F^{1} \neq \emptyset$. The converse holds by $(\mathrm{C})$.

Proposition 4.11. If $G$ is a finite subgraph of a graph $E$ and $S \subseteq R(E)$, there is a complete subobject $(F, T)$ of $(E, S)$ such that $F$ is finite, $G$ is a subgraph of $F$ and $C L_{K}(F, T)$ is a $K$-subalgebra of $C L_{K}(E, S)$. 
Proof. Following [3, Proposition 3.5], we define the graph $F$ and the set $T \subseteq R(F)$ as follows.

$$
\begin{gathered}
F^{0}=G^{0} \cup\left\{\mathbf{r}_{E}(e) \mid e \in E^{1}, \quad \mathbf{s}_{E}(e) \in G^{0} \cap S \text { and } \mathbf{s}_{E}^{-1}\left(\mathbf{s}_{E}(e)\right) \cap G^{1} \neq \emptyset\right\}, \\
F^{1}=G^{1} \cup\left\{e \in E^{1} \mid\right. \\
\left.\mathbf{s}_{E}(e) \in G^{0} \cap S \text { and } \mathbf{s}_{E}^{-1}\left(\mathbf{s}_{E}(e)\right) \cap G^{1} \neq \emptyset\right\}, \text { and } \\
T=S \cap\left\{v \in F^{0} \mid \mathbf{s}_{E}^{-1}(v) \cap F^{1} \neq \emptyset\right\} .
\end{gathered}
$$

With these definitions, $F$ is finite, $T \subseteq S$, and if $v \in S \cap F^{0}$ with $\mathbf{s}_{E}^{-1}(v) \cap F^{1} \neq \emptyset$, then $v \in T$ and $\mathbf{s}_{E}^{-1}(v) \cap G^{1} \neq \emptyset$ so $\mathbf{s}_{F}^{-1}(v)=\mathbf{s}_{E}^{-1}(v)$ by definition of $F^{1}$. Thus condition (C) holds. It is straightforward to see that axioms (V), (E1), (E2) and (CK1) are compatible in $C L_{K}(E, S)$ and $C L_{K}(F, T)$. If $v \in T$, then $\mathbf{s}_{F}^{-1}(v)=\mathbf{s}_{E}^{-1}(v)$ is finite. Thus, $\sum_{e \in \mathbf{s}_{F}^{-1}(v)} e e^{*}=$ $\sum_{e \in \mathbf{s}_{E}^{-1}(v)} e e^{*}=v$ so $(\mathrm{SCK} 2)$ is compatible as well. The inclusion of $(F, T)$ into $(E, S)$ induces the inclusion of the basis of $C L_{K}(F, T)$ into the basis of $C L_{K}(E, S)$ described in $\underline{3}$, Propositions 2.7 and 3.6]. This induces an embedding of $C L_{K}(F, T)$ into $C L_{K}(E, S)$.

In case when $S=R(E), E$ has an infinite emitter $v$ and $G$ is a subgraph consisting of $v$ with finitely many edges $v$ emits together their ranges, the complete subobject $(F, T)$ from Proposition 4.11 is such that $v \in R(F)$ but $v \notin T$. Thus $C L_{K}(F, T)$ is a $K$-subalgebra of $L_{K}(E)$ while $L_{K}(F)$ is not. Cases like this one make the consideration of Cohn-Leavitt algebras necessary in the proof of our next result, the main result of this section.

Theorem 4.12. The following conditions are equivalent.

(1) $L_{K}(E)$ is directly finite.

(2) $L_{K}(E)$ is finite.

(3) E is no-exit.

Proof. (1) trivially implies (2). (2) implies (3) by Proposition 4.3 ,

To show that (3) implies (1), assume that $x, y \in L_{K}(E)$ are such that $x u=u x=x$, $y u=u y=y$ and $x y=u$ for some idempotent $u$ of $L_{K}(E)$. If $x, y$, and $u$ are finite $K$-linear combinations of elements of the form $p_{i} q_{i}^{*}, i=1, \ldots, n$ for some paths $p_{i}$ and $q_{i}$, let $G$ be the subgraph of $E$ such that $G^{0}$ is the set of all vertices appearing in paths $p_{i}$ and $q_{i}, i=1, \ldots, n$, and $G^{1}$ is the set, possibly empty, of all edges of paths $p_{i}$ and $q_{i}$. Let $(F, T)$ be the complete subobject of $(E, R(E))$ generated by $G$ from Proposition 4.11, The graph $F$ is no-exit since $E$ is no-exit. If a vertex $v$ is in a cycle of $F$ then it emits a single edge both in $F$ and in $E$. Thus $v$ is regular and $\mathbf{s}_{E}^{-1}(v) \cap F^{1} \neq \emptyset$ so $v$ is in $T$ by definition of $F$ and $T$. This enables us to use Proposition 4.10 and to conclude that $C L_{K}(F, T)$ is directly finite. By construction $x, y$ and $u$ are elements of $C L_{K}(F, T)$ and so $u^{2}=u, x u=u x=x, y u=u y=y$ and $x y=u$ are relations in $C L_{K}(F, T)$ as well. Since $C L_{K}(F, T)$ is directly finite, these relations imply $y x=u$. The relation $y x=u$ then holds in $L_{K}(E)$ as well. So $L_{K}(E)$ is directly finite too.

Theorem 4.12 has the following corollary.

Corollary 4.13. The following conditions are equivalent.

(1) $C L_{K}(E, S)$ is directly finite.

(2) $C L_{K}(E, S)$ is finite.

(3) $E$ is no-exit and vertices of every cycle are in $S$.

In particular, a Cohn path algebra $C_{K}(E)$ is (directly) finite if and only if $E$ is acyclic. 
Proof. (1) trivially implies (2) and (2) implies (3) by Proposition 4.10. To show that (3) implies (1), note that (3) implies that the graph $E_{S}$ is no-exit. Thus $L_{K}\left(E_{S}\right)$ is directly finite by Theorem 4.12 and then so is $C L_{K}(E, S) \cong L_{K}\left(E_{S}\right)$.

The equivalence of conditions (1), (2) and (3) with $S=\emptyset$ shows the last sentence.

In the final part of the paper, we focus on relations between the following three conditions.

(1) $L_{K}(E)$ is locally noetherian.

(2) $L_{K}(E)$ admits a faithful trace.

(3) $L_{K}(E)$ is directly finite.

Recall that a ring $T$ is locally left (right) noetherian if for every finite set $F$ of $T$, there is an idempotent $e \in T$ such that $e T e$ contains $F$ and $e T e$ is left (right) noetherian. By [1, Theorem 3.7], a Leavitt path algebra is locally left noetherian if and only if it is locally right noetherian and we simply say it is locally noetherian in this case. Recall also that an infinite path of a graph is a sequence of edges $e_{1} e_{2} \ldots$ such that $\mathbf{r}\left(e_{i}\right)=\mathbf{s}\left(e_{i+1}\right)$ for all $i=1,2, \ldots$. An infinite path $p$ is an infinite sink if it has no cycles or exits. An infinite path $p$ ends in a sink if there is $n \geq 1$ such that the subpath $e_{n} e_{n+1} \ldots$ is an infinite sink, and $p$ ends in a cycle if there is $n \geq 1$ and a cycle $c$ of positive length such that the subpath $e_{n} e_{n+1} \ldots$ is equal to the path $c c \ldots$ [1, Theorem 3.7] asserts that the following conditions are equivalent for every graph $E$.

(1) $L_{K}(E)$ is locally noetherian.

(2) $E$ is a no-exit graph such that every infinite path ends either in a sink or in a cycle.

Proposition 4.2. Theorem 4.12, and [1, Theorem 3.7] infer the implications and equivalences in the diagram below.

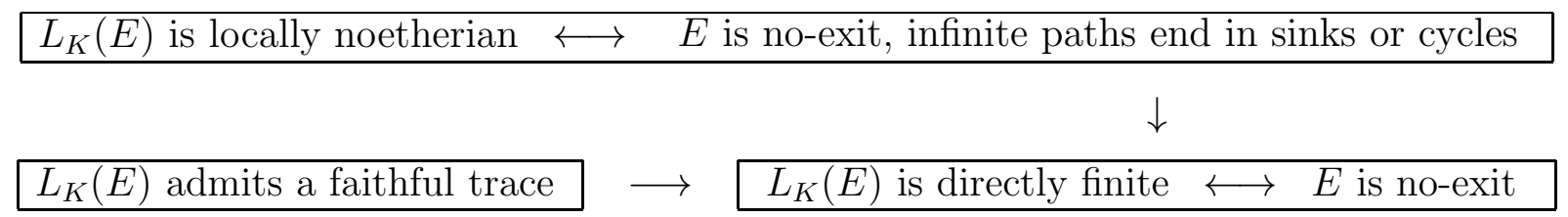

The next three examples show that both implications are strict and the two conditions " $L_{K}(E)$ is locally noetherian" and " $L_{K}(E)$ admits a faithful trace" are independent.

Example 4.14. If $E$ is any no-exit graph which has an infinite path not ending in a sink or a cycle, then $L_{K}(E)$ is directly finite and not locally noetherian. For example, the graph $E$ below has this property.

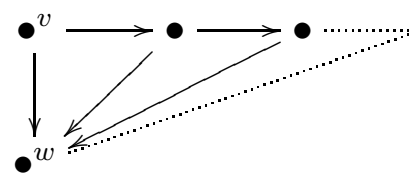

There is no faithful $\mathbb{C}$-valued graph trace on $E$ since there are infinitely many paths from $v$ to $w$ forcing $\delta(w)$ to be zero for any positive graph trace $\delta$ on $E$. This demonstrates that a directly finite Leavitt path algebra may not admit a faithful trace.

Example 4.15. Let $E$ be the graph with two vertices $v$ and $w$ and infinitely many edges from $v$ to $w$ as represented below. The Leavitt path algebra of this graph over any field is locally noetherian since $E$ satisfies the required graph-theoretic condition. However, there 
is no faithful trace on $L_{\mathbb{C}}(E)$ (nor $L_{\mathbb{R}}(E)$ as noted also in [12, Example 35]) since there are infinitely many paths from $v$ to $w$.

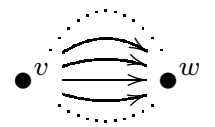

Example 4.16. The graph $E$ represented below is such that there is an infinite path which does not end in a sink or a cycle so $L_{K}(E)$ is not locally noetherian for any field $K$.

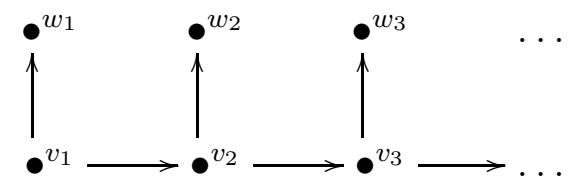

On the other hand, there is a faithful, $\mathbb{C}$-valued graph trace given by

$$
\delta\left(v_{n}\right)=\frac{1}{2^{n-1}} \quad \text { and } \quad \delta\left(w_{n}\right)=\frac{1}{2^{n}} \quad \text { for } n=1,2, \ldots
$$

The graph trace $\delta$ extends to a faithful trace by Theorem 3.6.

It is interesting to note that both conditions " $L_{K}(E)$ is locally noetherian" and " $L_{K}(E)$ is directly finite" have been characterized by graph-theoretic conditions. We wonder if a graph-theoretic characterization can be found for the condition " $L_{K}(E)$ admits a faithful trace" if $K$ is positive definite. If $E$ is a row-finite graph in which every infinite path ends in a sink or a cycle, [12, Theorem 33] shows that $L_{K}(E)$ admits a faithful trace if and only if $E$ is no-exit. However the graph $E$ from Example 4.16 does not fall under the class of graphs covered by [12, Theorem 33] and $L_{\mathbb{C}}(E)$ admits a faithful trace. So, we wonder if a general graph-theoretic characterization is possible. More precisely, we propose the following.

Open Problem 4.17. Find a graph-theoretic condition on $E$ which is equivalent to the condition that the Leavitt path algebra $L_{K}(E)$ over a positive definite field $K$ admits a faithful trace.

A similar question was also raised in [14] for graph $C^{*}$-algebras. [14, Lemma 3.5 and Corollary 3.7] show that the following condition is necessary for a row-finite graph $E$ to admit a $\mathbb{C}$-valued faithful graph trace.

(1) There are finitely many paths from any vertex to any other vertex, an infinite sink or a loop.

If $E$ is row-finite, [14, Proposition 3.8] lists the following condition as sufficient.

(2) There is a finite upper bound for the number of paths from any vertex to any other vertex, an infinite sink or a loop and every infinite path ends in a loop or a sink.

Condition (2) is not necessary as the following example shows.

Example 4.18. Let $E$ be the graph with vertices $v$ and $w_{n}, n=1,2, \ldots$, and $n$ edges from $w_{n}$ to $v$ for every $n$. In the diagram below, the numbers next to the arrows indicate the 
number of edges from vertices $w_{n}$ to $v$.

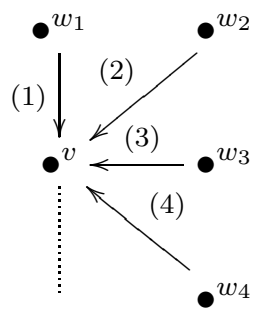

The graph $E$ is row-finite, it does not satisfy condition (2), and there is a faithful, $\mathbb{C}$-valued graph trace on $E$ given by

$$
\delta(v)=1 \quad \text { and } \quad \delta\left(w_{n}\right)=n \text { for } n=1,2, \ldots
$$

In fact, if $K$ is any positive definite field, the conditions above define a faithful, $K$-valued graph trace on $E$.

Acknowledgments. The author is grateful to Zachary Mesyan for his valuable suggestions on an early outline of the manuscript and, in particular, for pointing out a gap in the proof of Theorem 3.4 from that version of the manuscript.

\section{REFERENCES}

[1] G. Abrams, G. Aranda Pino, F. Perera and M. Siles Molina, Chain conditions for Leavitt path algebras, Israel J. Math. 165 (2008), 329-348.

[2] G. Abrams, M. Tomforde, Isomorphism and Morita equivalence of graph algebras, Trans. Amer. Math. Soc., 363 (2011), 3733 - 3767.

[3] P. Ara, K. R. Goodearl, Leavitt path algebras of separated graphs, J. Reine Angew. Math. 669 (2012), $165-224$.

[4] P. Ara, M.A. Moreno, E. Pardo, Nonstable K-theory for graph algebras, Algebr. Represent. Theory, 10 (2007), 157-178.

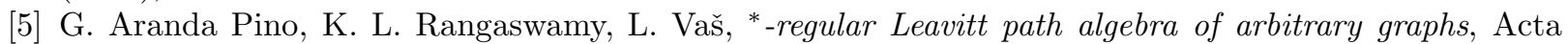
Math. Sci. Ser. B Engl. Ed. 28 (5) (2012), 957 - 968.

[6] G. Aranda Pino, L. Vaš, Noetherian Leavitt path algebras and their regular algebras, Mediterr. J. Math., 10 (4) (2013), 1633 - 1656.

[7] T. Bates, J. H. Hong, I. Raeburn, and W. Szymański, The ideal structure of $C^{*}$-algebras of infinite graphs, Illinois J. Math 46 (2002), 1159-1176.

[8] T. Bates, D. Pask, I. Raeburn, W. Szymanski, The $C^{*}$-algebras of row-finite graphs, New York J. Maths 6 (2000), 307-324.

[9] S. K. Berberian, Baer *-rings, Die Grundlehren der mathematischen Wissenschaften 195, SpringerVerlag, Berlin-Heidelberg-New York, 1972.

[10] M. Johnson, The graph traces of finite graphs and applications to tracial states of $C^{*}$-algebras, New York J. Math. 11 (2005), 649-658.

[11] R. V. Kadison, J. R. Ringrose, Fundamentals of the theory of operator algebras, volume 1: Elementary theory, Pure and Applied Mathematics Series 100, Academic Press, London-New York, 1983.

[12] Z. Mesyan, L. Vaš, Traces on Semigroup Rings and Leavitt Path Algebras, Glasgow Mathematical Journal, in print.

[13] P. S. Muhly, M. Tomforde, Adding tails to $C^{*}$-correspondences, Doc. Math. 9 (2004), 79-106.

[14] D. Pask, A. Rennie, The noncommutative geometry of graph $C^{*}$-algebras I: The index theorem, J. Funct. Anal. 233 (1) (2006), 92-134.

[15] M. Tomforde, Uniqueness theorems and ideal structure for Leavitt path algebras, J. Algebra 318 (2007), 270-299.

[16] M. Tomforde, Real Rank Zero and Tracial States of $C^{*}$-Algebras Associated to Graphs, arXiv, math. OA/0204095 v3, 2002. 
Lia Vaš, Department of Mathematics, Physics and Statistics, University of the Sciences, PhiladelPhia, PA 19104, USA

E-mail address: 1.vas@usciences.edu 